\title{
Functional and Phenotypic Analysis of Two-Day Monocyte-Derived Dendritic Cells Suitable for Immunotherapy Purposes
}

\author{
David Andrés Bernal-Estévez ${ }^{1,2, \#, ~ D i a n a ~ R o c i o ~ T o v a r ~ M u r i l l o ~}{ }^{1,2, \#}$, and Carlos Alberto Parra- \\ López ${ }^{2 *}$ \\ ${ }^{1}$ Immunology and Clinical Oncology Research Group (GIIOC). Fundación Salud de los Andes, Bogotá-Colombia South-America. \\ ${ }^{2}$ Immunology and Translational Medicine Research Group, Universidad Nacional de Colombia, Graduated School in Biomedical Sciences. Department of \\ Microbiology. Carrera 30 Calle 45, Bogotá-Colombia South-America. \\ ${ }^{\#}$ Both authors contribute equally to this work.
}

Received: November 07, 2016; Accepted: November 17, 2016; Published: December 19, 2016

*Corresponding author: Carlos Alberto Parra-López, Universidad Nacional de Colombia, Facultad de Medicina, Departamento de Microbiología oficina, Carrera 30 Calle 45-03, Bogotá, Colombia, Tel:+57 13165000 x 15016; Fax: (+57 1) 3165000 x 15024; E-mail: caparral@unal.edu.co

\section{Abstract}

Background: Optimize the generation of clinical-grade monocyte-derived dendritic cells (DC) suitable for cancer immunotherapy affordable to cancer patients worldwide remains a formidable challenge. Although vaccination with DC pulsed with tumor-associated antigens (TAA) has been thoroughly evaluated in Caucasian individuals, the immunogenicity of DCs in Colombian individuals is yet to be explored.

Methodology: Mononuclear cells obtained from buffy coats of healthy Colombian volunteers were used to compare the phenotype and proficiency as Antigen Presenting Cells (APC) of matured DCs obtained in two vs. seven days from monocytes purified either by adherence to plastic or by negative selection used fresh or after cryopreservation. Furthermore, the functional capacity of two types of $2 \mathrm{~d}$-DCs maturated with two different cytokine-cocktails: Standard (2d-stDCs) or Type I Alpha (2d-aDCs), to prime or boost TAA specific CD8+ T cells and to stimulate Th1 stem cells and follicular T helper CD4+ T-cells (TFH), was examined.

Results: Both 2d-stDCs and 2d-aDCs exhibited a maturation profile similar to standard DCs obtained in seven days. Despite both types of DCs were efficient in stimulating antigen specific CD4+ and CD8+ T cells, only $2 \mathrm{~d}-\mathrm{aDCs}$ secreted high amounts of IL-12p70 and fostered more efficiently than $2 \mathrm{~d}$-stDCs the expansion of stem memory Th1, TFH cells and effector TAA specific CD8+ T cells.

Conclusions: Two-day derived DCs are phenotypically and functionally equivalent to seven day derived DCs and are suitable for cancer immunotherapy in Colombian individuals.

Key Words: Dendritic cells; T Lymphocytes; Flow Cytometry; Immunotherapy; HLA-A*0201-restricted Tumor-Associated Antigen.

\section{Abbreviations}

DCs: Dendritic Cells; 2d-DCs: two-day monocyte-derived DCs; 7d-stDCs: seven-day monocyte-derived DCs; iDCs: immature DCs; PBMCs: Peripheral Blood Mononuclear Cells; FC: Flow Cytometry; DC-SIGN: Dendritic cell-specific ICAM-3 grabbingnonintegrin (CD209); CFSE: Carboxy Fluorescein Succinimidyl ester; TAAs-A2: Tumor associated antigens.

\section{Introduction}

Since the initial description of dendritic cells (DCs) more than 40 years ago [1], their role in $\mathrm{T}$ cell mediated immunity in particular in anti-tumor immunity have been deeply studied [2]. The clinical use of autologous DCs for therapy in cancer patients have shown that DCs are a secure therapeutic strategy that elicit variable clinical responses with low adverse effects in treated patients[3]. This strategy aims the recovery of the immune system unresponsiveness promoted by the tumor microenvironment that ends up with the reestablishing of the anti-tumor capacity of cytotoxic T cells (CTLs) [4-10]. Characterizing the phenotype of monocyte-derived DCs after maturation continue being a valuable tool to examine in vitro the potential benefits of these cells in generating protective immunity against tumors in cancer patients.

In humans, there are two groups of primary DCs, the myeloid DCs characterized by the expression of CD11c and CD33, and lymphoid derived or plasmacytoid DCs which express CD123 and CD45RA. While the number of myeloid DCs in peripheral blood or infiltrating DCsintumors is decreased, the number of plasmacytoid DCs is not altered. Robust evidence suggests that myeloid DCs in cancer patients have an immature phenotype $[11,12]$ that induce immune tolerance within tumor microenvironment $[13,14]$. That DCs isolated from patients recover responsiveness to maturation stimuli in vitro or that DCs from normal individuals express an immature phenotype when exposed to tumor cells supernatant, suggests that in cancer patients a wide variety of stimuli secreted by tumors hampers functionality of myeloid DCs[15]. Therefore, vaccines based on DCs has been actively explored for the last several years to surmount both the poor presentation of tumor antigens by tumor cells likewise the deficient performance as APCs of primary DCs in cancer patients [15].

The ability of DCs to activate T cells capable of recognizing and destroying tumor cells in an antigen-specific manner has been demonstrated in clinical protocols carried out in cancer patients 
in which DCs pulsed with tumor antigens has been used as vaccines [16]. It has been observed that this type of vaccine is safe and more tolerable than other treatments such as chemotherapy or radiation therapy [3], with variable clinical and immunological responses [5, 17-19]. The clinical evidence suggests that immune stimulation of cancer patients with autologous DCs pulsed in vitro with tumor antigens is possible and that the use of this class of therapeutic vaccines in a variety of invasive tumors opens the possibility of prolonging the life expectancy of treated patients while improving their quality of life.

Because DCs are in a very low amount in peripheral blood, during the last decade it has been described several methods for their generation in vitro from peripheral blood monocytes cultured with IL-4 and GM-CSF and maturated with various combinations of pro-inflammatory cytokines and/or Toll-Like Receptors (TLRs) agonists [20]. Most of clinical trials conducted to date have used monocyte-derived DCs generated in seven days (7d-DCs): five days in the presence of GM-CSF and IL-4 followed by the addition of the maturation stimuli for two days. Additionally, there are some reports proposing the generation of DCs in a shorter period of time: DC induction from monocytes treated with GM-CSF and IL-4 for 24 hours is followed by 24 hours incubation with a pro-inflammatory stimulus ( $2 \mathrm{~d}-\mathrm{DCs})$, a timing that allows to achieve competent DCs that elicits antigenspecific Th1 immune responses[21, 22]. Translation of this modification in timing into the clinic would make DC production less laborious and will drop the costs of mass production of DCs, two features of DC production that must be considered to make DC-based immunotherapy more accessible to cancer patients in undeveloped countries. Finally, a newly described combination of cytokines for maturation of DCs that includes type I interferons and TLR ligands [23] that induces mature DCs denominated Type I alpha DCs or alpha-DCs, that are characterized by high production of IL-12 and the efficient activation of CD8+ T cells against tumors.

Given the importance of implementing new alternatives for the management of cancer in Colombia, in this work, we evaluated yield of production, phenotypic and functional characteristics of matured 2d-DCs from healthy Colombian individuals obtained through two different combinations of maturation cytokines. Our results demonstrate that DCs derived in two days, have a clear maturation phenotype and the capacity of activating $\mathrm{T}$ cells (both cytotoxic and follicular helper - TFH cells), suggesting that 2d-DCs are suitable to be used in Colombian individuals for cancer immunotherapy.

\section{Materials and Methods}

Patients and monocyte enrichment: This study was approved through the Ethics Committee of the Medical School Universidad Nacional de Colombia (CE-14, 9 August 2012, Act. 107). All participants provided informed consent before the blood samples were collected. Heparinized blood samples $(60 \mathrm{~mL})$ were obtained from healthy volunteers or latent Mycobacterium tuberculosis (M. tb.) infected (LTBI) donors (QuantiFERON TB Gold test in tube positive and Tuberculin Skin Test positive) and a blood sample $(20 \mathrm{~mL})$ was obtained from a breast cancer patient diagnosed with ductal breast cancer (HLA-A*02:01) before chemotherapy. PBMCs were purified using density gradient ficoll and cryo-preserved in freezing medium (50\% RPMI + 40\% FCS $+10 \%$ DMSO). Monocytes were enriched after the adherence to plastic for 2 hours or by negative selection (RosetteSep STEMCELL Technologies).

Dendritic cell generation: Immature DCs (iDCs) were differentiated from purified monocytes or total PBMCs using $1000 \mathrm{UI} / \mathrm{mL}$ of GM-CSF and $750 \mathrm{UI} / \mathrm{mL}$ of IL-4 (Cellgenix Germany) for 24 hours (for $2 \mathrm{~d}$-aDCs or $2 \mathrm{~d}$-stDCs generation) based on the methodology described by Dauer et al.[21] or 5 days (for 7d-stDCs generation) as described by Jonuleit et al. [24]. iDCs were matured using two different cytokine cocktails: the standard cocktail, containing IL-1 $\beta$, IL-6, TNF- $\alpha$ (all from Cellgenix) and PGE2 (Sigma-Aldrich) for 24 (2d-stDCs) or 48 hours (7d-stDCs); or the Type I alpha cocktail, containing IFN- $\alpha$ (Intron-A; Schering Plough Corp., Kenilworth, NJ), IFN- $\gamma$ (R\&D Systems), Poly I:C (Sigma-Aldrich), TNF- $\alpha$, IL-6, and IL-1 $\beta$ (Cellgenix - Germany) for 24 hours (2d-aDCs) as previously described by Mailliard et al.[23]. All cells (fresh or after cryopreservation in liquid nitrogen) were cultured in AIM-V medium (Life Technologies).

Mixed Leukocyte Reaction (MLR) and proliferation assays: Highly purified CD4+ T cells by magnetic beads system (Miltenyi Biotec - Germany) were obtained from PBMCs of healthy donors and stained with $2 \mu \mathrm{M}$. CFSE (Life technologies); labeled cells were stimulated with autologous $2 \mathrm{~d}$-stDCs or $7 \mathrm{~d}$-stDCs, pulsed with tetanus toxoid or stimulated with $10 \mu \mathrm{M}$ phytohemagglutinin (PHA)-M (Sigma-Aldrich). After 6 days of cell culture, CD4+ T cells were labeled with anti-CD69 (BD). For MLR, purified CD4+ T cells from three different donors were labeled with CFSE and co cultured with allogeneic 2d-stDCs for 6 days, after harvesting the cell T cells were labeled with antiCD69 and analyzed by flow cytometry in a FACS Aria II (BD).

T cells stimulation and cytotoxicity assays: Two different $T$ cell stimulation methods were used [Figure S1]. Total PBMCs were enriched with $2 \mathrm{~d}$-stDCs or $2 \mathrm{~d}$-aDCs based on the methodology of Martinuzzi et al.[25]. Briefly, PBMCs were cultured with IL-4 and GM-CSF (as described above) for 24 hours in the presence of $5 \mu \mathrm{M}$ of Tumor-Associated Antigens (TAAs) Her2/neu, Melan-A (MART-1), NY-ESO-1, Telomerase, $M$. $t b$. peptides as described by Goletti et al.[26], purified ESAT- 6 protein or $M$. $t b$. H37Rv sonicate, and L. major peptide as control. After 24 hours the iDCs were subsequently maturated using either $2 \mathrm{~d}$-stDCs or $2 \mathrm{~d}$-aDCs maturation cocktails, with or without the addition of $5 \mu \mathrm{M}$ of the corresponding peptide(s) for 6 days [Figure S1A]. The second method is based on the methodology of Moser et al.[27]; briefly, frozen PBMCs were sorted (FACS Aria II - BD) into three different populations, CD14+ (monocytes), CD4+ and CD8+ T cells, using the BD FACS Aria II System (BD Biosciences). The monocytes were differentiated into $2 \mathrm{~d}$-stDCs or $2 \mathrm{~d}-\mathrm{aDCs}$, pulsed or unpulsed with corresponding peptides or proteins ( $5 \mu \mathrm{M}$ each) and subsequently cultured with purified CD4+ or CD8+ T cells for 14 days at a ratio of 50:1 (T cell: DCs) in AIM-V culture media (Life Technologies), for CD8+ T cell culture $30 \mathrm{UI} / \mathrm{mL} \mathrm{IL-2}$ was added. After priming, CD4+ or CD8+ T cells were stimulated with 
corresponding peptide-pulsed 2d-stDCs or $2 \mathrm{~d}-\mathrm{aDC}$ and cultured for 6 additional days [Figure S1B] For cytotoxicity assays, CD8+ $\mathrm{T}$ cells from healthy donors were stimulated as described above, and labeled with CD107a or CD107b for 12 hours before flow cytometry analysis.

Peptide synthesis: All peptides were generated through solid phase peptide synthesis $\left(21^{\text {st }}\right.$ Century Biochemicals, CPC Scientific and in the Fundación Instituto de Inmunología de Colombia - FIDIC), with high purity $>85 \%$. The lyophilized peptides were dissolved in DMSO and diluted in PBS to a working concentration of $2 \mathrm{mM}$ each.

Flow cytometry and cytokine quantification: The DCs were labeled with CD80 PE, CD83 PE-Cy5, CCR7 FITC, HLA-DR APC, CD14 Alexa Fluor 700, CD209 FITC, CD123 PE or CD11c APC (all from BD). Purified T cells or total PBMCs were stained with CD3 Pacific Blue, CD4 ECD (Beckman coulter) or CD8 PE-Texas Red (eBiosciences), CD45RO FITC, CD45RA PE-Cy5, CD62-L PE (BD), CD154 APC (eBiosciences), CD95 APC, or CCR7 FITC (R\&D Systems). The intracellular staining of IFN $-\gamma$ PE, TNF- $\alpha$ APC, and IL-2 PE (BD biosciences) was performed using cytofix/cytoperm and perm/wash reagents from BD Biosciences. Biotinylated HLA-A*02:01 tetramers were synthesized at the Lawrence Stern Laboratory, University of Massachusetts Medical school by CP, Melan-A tetramer was kindly gifted by Professor Pedro Romero, Ludwig Cancer Research Institute - Switzerland, and the tetramers were labeled with streptavidin-PE (Invitrogen) at a 4:1 molar ratio before use. Cytokine secretion (TNF- $\alpha$, IFN- $\gamma$, IL-6 or IL-12p70) was measured in the culture supernatants using human Th1/Th2 and Inflammatory CBA kits (BD Biosciences). The samples were acquired using the FACS Aria II System at the Universidad Nacional de Colombia - Medical School. The flow cytometry data were exported in FCS format v3 and analyzed using Flow Jo software (Treestar Inc.). The graphics were generated using Prism v5 software (Graph Pad).

Microscopy: The images of iDCs, 2d-aDCs and 2d-stDCs were obtained using an inverted microscope (Nikon Eclipse TS100) coupled to a digital camera (Canon Powershot S120) at 20x magnification. The pictures were cropped in Photoshop CS5 (Adobe).

Statistical analysis: The data was analyzed by Two-way ANOVA with Bonferroni posttest to compare the mean between groups, and Mann-Whitney test for non-parametric analysis between immature and mature cells. $\mathrm{p}$ value was significant if below $0.05\left(^{*}\right), 0.01\left(^{* *}\right), 0.001\left(^{* * *}\right)$ or $0.0001\left(^{* * *}\right)$. Data was analyzed in Prism v5 software (Graph Pad).

\section{Results}

Two-day monocyte-derived dendritic cells (2d-DCs) have similar characteristics than Dendritic cells derived in seven days (7d-DCs)

The optimization process of generating DCs involves the reduction of cultivation time, usually DCs are generated within 7 days, but reducing the time to two-day culture, reduces costs, increases cell viability and the total number of DCs that can be recovered to be used in immunotherapy. Initially, we compared side by side the immune phenotype and functional characteristics of immature DC (iDC) and after treatment for 24 or 48 hours in the presence of the standard cytokine cocktail (IL-1 $\beta$, IL-6, TNF- $\alpha$, and PGE2) based on the methodology described by Dauer et al.[21,28]. Monocytes purified from healthy donors were cultured for 24 hours or 5 days in the presence of IL-4 (1000 IU $/ \mathrm{mL})$ and GM-CSF (750 IU/mL). Subsequently, to induce maturation of $2 \mathrm{~d}$-stDCs and $7 \mathrm{~d}$-stDCs, the iDCs were cultured in the presence of the maturation cocktail containing IL- $1 \beta$, IL-6, TNF- $\alpha$ and PGE2 [10]. As shown in Figure 1A, changes in cell morphology were evident by direct observation under microscope, initially noted that $7 \mathrm{~d}$-DCs have a larger size compared with the size of 2d-DCs despite the maturation state, however, after 24 hours of maturation, DCs change from a round shape to present elongated dendrites in $2 \mathrm{~d}$-stDCs, and present multiple dendrites in $7 \mathrm{~d}$-stDCs. This difference of size and complexity was confirmed by Flow Cytometry (FC) using SSC-A vs. FSC-A analysis [Figure 1B], where $7 \mathrm{~d}$-DCs have the greatest distribution of light dispersion in contrast to 2d-stDC or monocytes. Subsequently, multi-parametric FC was used to compare the expression of CD83, CD209, CD14, CCR7, CD80, HLA-DR, and CD86 in iDCs, and mature DCs $2 \mathrm{~d}$-stDCs and 7d-stDCs. The standard cocktail of cytokines in $7 \mathrm{~d}$-stDCs clearly induces a maturation phenotype as expected [Figure 1C], with increased expression of CD80, CD83, CCR7, CD86 and HLA-DR and decreased expression of CD14 and CD209. This phenotype was consistent with previous studies $[29,30]$. In particular, treating iDCs with maturation stimuli for 24 hours generates $2 \mathrm{~d}$-stDCs with a phenotype very similar to that of $7 \mathrm{~d}$-stDCs maturation in terms of increase, CD80, CD86, CCR7, CD83, and HLA-DR and decreasing of CD209 and CD14 expression [Figure 1C]. These results suggest that the time reduction necessary to generate mature DCs in vitro does not affect the phenotype of mature DCs.

\section{2d-stDCs have a similar mature phenotype in compari- son to $7 \mathrm{~d}$-stDCs regardless of monocyte purification system}

Despite several procedures available to obtain monocytes from peripheral blood such as (i) positive or negative selection of CD14 cells (either by flow cytometry or magnetic beads) and (ii) cell adhesion to plastic, plastic adherence of monocytes has been the procedure most widely used to produce DCs from monocytes in vitro. During the optimization process of DC production, we compared two procedures to obtain monocytes from peripheral blood, first, by negative selection and second, by adherence to plastic that is a low-cost procedure and a rather "standard method" for monocyte enrichment. Additionally, we evaluated if the cryopreservation of purified monocytes compared to freshly isolated monocytes (isolated by negative selection or adherence to plastic) did affect maturation of DCs.

We evaluated the effect of pro-inflammatory cytokines standard cocktail to induce mature DCs (Figure 2). Initially, we evaluate the phenotype of DCs derived from monocytes purified by negative selection, as expected the pro-inflammatory cytokines induce a significant increase in the expression of CCR7, 
A

B
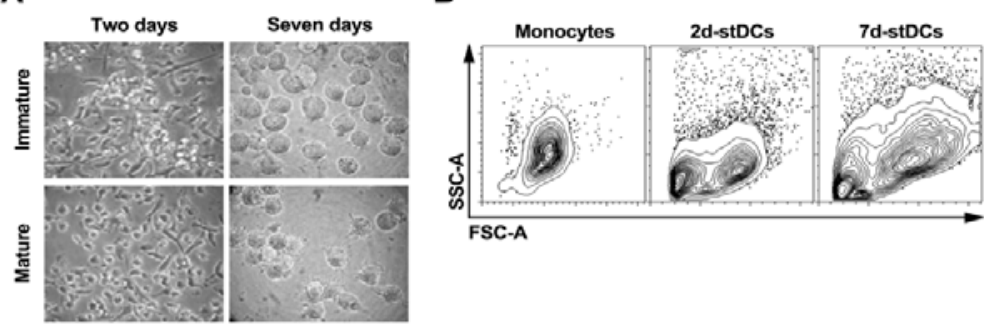

C

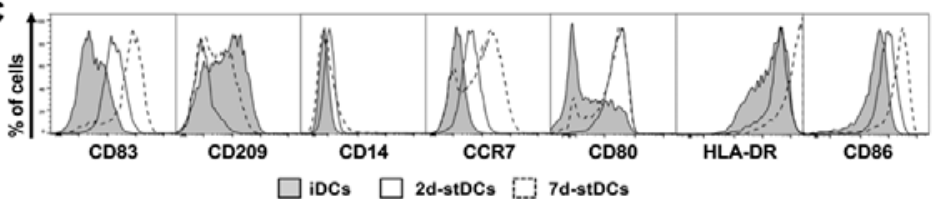

Figure 1: Morphological and phenotypic differences between dendritic cells matured in seven days (7d) and two days (2d). A. Microscopic view (40X) of the DC-derived in vitro from purified monocytes, mature or immature $2 \mathrm{~d}$, and $7 \mathrm{~d}$ ( $2 \mathrm{~d}$-iDCs, $7 \mathrm{~d}$-iDC). B. Contour plots of FSC-A vs. SSC-A of purified monocytes, dendritic cells matured in $2 \mathrm{~d}$ and $7 \mathrm{~d}$-DCS (from left to right respectively). C. Histograms representing the expression of maturation markers, CD83, CD209, CD14, CCR7, CD80, HLA-DR, and CD86 on immature dendritic cells (gray histogram), two days mature DCs (empty histogram) and seven days mature DCs (Histogram with dashed line).

CD86, CD83, and CD80 with the expected reduction of CD209 in $2 \mathrm{~d}$-stDCs and 7d-stDCs [Figure 2, panels A and E, respectively]. When we evaluate the phenotype of DCs derived in two and seven days from adherent cells suitable for clinical use, we found that the purification method does not affect the phenotype in mature DCs. Interestingly, 2d-stDCs have a more pronounced difference between immature and mature cells than $7 \mathrm{~d}$-stDCs [Figure 2, panels B and F, respectively]. Finally, it is important to compare the effect of monocyte cryopreservation in the phenotype of mature DCs, to evaluate this, we analyze the same phenotype as described before in freshly isolated and cryopreserved monocytes in either two days and seven days DCs (Figure 2), panels C, D, G, and $\mathrm{H}$. The phenotype found in these cells evidence that the cryopreservation of monocytes does not affect the capacity of monocytes to respond to pro-inflammatory cytokines, even more, $2 \mathrm{~d}$-stDCs have a more significant difference between immature and mature DCs compared to $7 \mathrm{~d}$-stDCs. These results also show that the quantification of HLA-DR and CD14 are not suitable for maturation markers of $2 \mathrm{~d}$-stDCs or $7 \mathrm{~d}$-stDCs. Finally, the mechanism for obtaining and preservation of monocytes does not affect their ability to respond adequately to the cocktail of cytokines.

Based in FDA approved Sipuleucel-T (Provenge ${ }^{\circledR}$ ) that consist of a fusion of GM-CSF with the Prostatic Acid Phosphatase (PAP) specific antigen of prostate cancer, used in total cells to induce antigen presentation by Antigen Presenting Cells (APC) thanks to the function of GM-CSF, we wanted to evaluate the advantages of inducing the differentiation and maturation of DCs from total PBMC without prior purification process of monocytes using a similar approach to that described by Martinuzzi et al., who evaluated the ability of IL-4 and GM-CSF followed by a maturation stimulus to amplify memory $\mathrm{T}$ cells in total PBMC [25]. Using two-days DCs, we compared the expression of CCR7,
CD80, CD83, and CD14, in CD11c+ cells in adherent and nonadherent fraction, exposed or not to the cocktail of cytokines [Figure S2], after collecting the cells we found that in the nonadherent fraction there are cells that have a phenotype of mature DCs with similar phenotype to cells that remain attached to plastic [Figure S2].

Finally, when we analyze the phenotype of 2d-stDCs in comparison to immature DCs, our results confirm that $2 \mathrm{~d}$-stDCs [Figure 2D] have a similar phenotype compared to $7 \mathrm{~d}$-stDCs [Figure $2 \mathrm{H}$ ]. This phenotype is characterized by a marked reduction of CD209 and increased expression of CCR7, CD80, and CD83 in 2d-stDCs. Despite differences in the morphology of DC [Figure 1], we found that DCs derived in two days, have a more pronounced difference between immature and mature DCs compared to DCs derived in seven days.

\section{Multiparametric analysis of DCs maturation markers confirms a similar phenotype between $2 \mathrm{~d}$ - and 7d-stDCs}

The study of DCs phenotype includes the expression of different profiles that includes up regulation of co-stimulatory and MHC class II molecules, chemokine receptors, among others, that make the definition of a mature DC difficult to compare between studies. In order to determine the value of the analysis of one or more maturation markers in the phenotype of $2 \mathrm{~d}$ - and $7 \mathrm{~d}$-stDCs we performed a multivariable analysis of two or more parameters to define the mature phenotype of DCs. Initially, we compared the phenotype of immature and 7d-stDCs to establish the combination that describes the best difference between immature and mature DC. Notably, combinations of three parameters that includes CD80, CD209 negative, and HLA-DR or two parameters: CCR7 and CD83 or CD83 and CD86 expression, exhibited the most significant difference [Figure 3A]. The same 
analysis process was also done with $2 \mathrm{~d}$-stDCs; In this case, most combination of markers (including two, or four parameters simultaneously), showed significant differences between mature and immature DCs [Figure 3B]. Finally, when comparing mature $2 \mathrm{~d}$-stDCs with $7 \mathrm{~d}$-stDCs [Figure 3C], we evidenced that most of the combinations of markers (except for CD80 and CD209 negative cells) were not statistically different between these two types of DCs suggesting that monocyte derived DCs obtained in either two and or seven days have a very similar maturation phenotype.

\section{Mature 2d-stDCs are capable of inducing specific T cell activation and proliferation}

Based on the DCs phenotype obtained in 2d-stDCs we assess the capacity of these cells to induce $T$ cell responses. To achieve this, mature $2 \mathrm{~d}$-stDCs from healthy individuals vaccinated against tetanus were pulsed with Tetanus Toxoid (TT) recombinant protein $(5 \mu \mathrm{M})$ and PHA-M $(5 \mu \mathrm{M})$ was added to a control culture

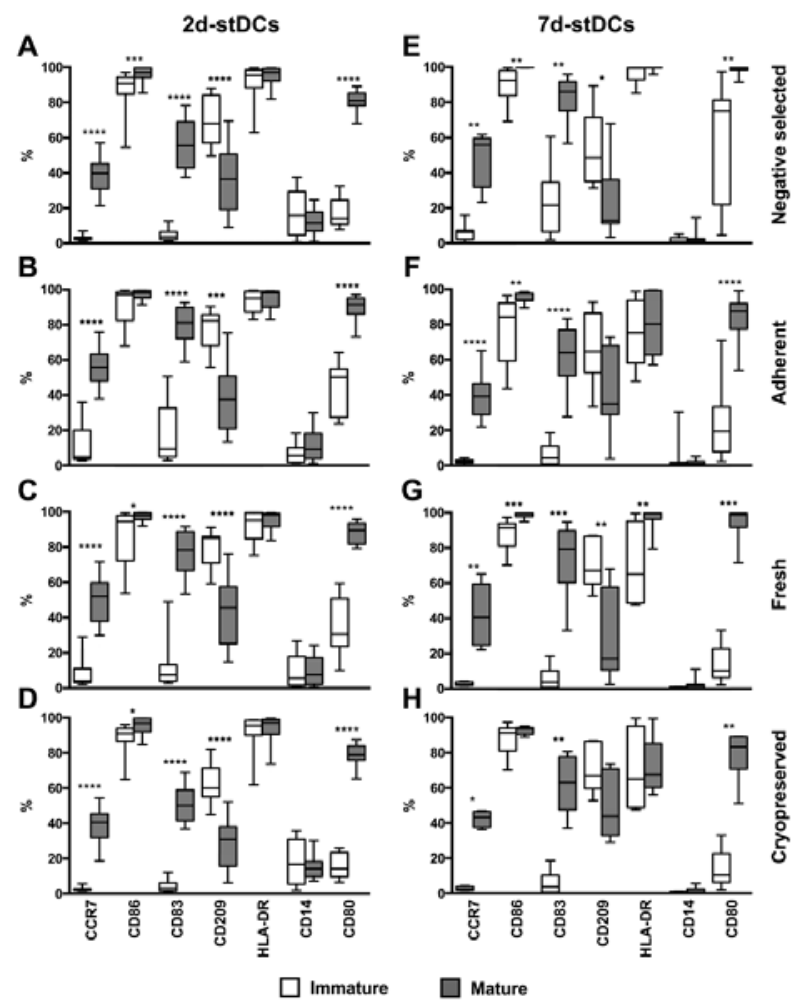

Figure 2: Monocyte collection system and preservation system do not affect the generation of dendritic cells. Comparative expression percentage by flow cytometry analysis of CCR7, CD86, CD83, CD209, HLA-DR, CD14 and CD80 in immature (white box) and mature DCs (gray box) derived in two or seven days (left and right columns respectively). Panel $A$ ( $n=18$ vs. 18 ) and $E(n=11$ vs 11$)$, corresponds to DCs derived from negative selected monocytes; B ( $n=9$ vs. 14) and F ( $n=7$ vs. 7), DCs derived from adherent monocytes; $C$ ( $n=15$ vs. 20) and $\mathrm{G}(\mathrm{n}=6$ vs.12), DCs derived from fresh monocytes; $\mathrm{D}(\mathrm{n}=12 \mathrm{vs.12})$ and $\mathrm{H}$ ( $n=6$ vs. 6), DCs derived from cryopreserved monocytes. Data showed in box and whiskers (top and bottom whiskers $90 \%$ and $10 \%$ ), the comparison between immature and mature DCs done with Mann-Whitney test ${ }^{*} \mathrm{p}<0.05,{ }^{* *} \mathrm{p}<0.01,{ }^{* * *} \mathrm{p}<0.001,{ }^{* * * *} \mathrm{p}<0.0001$. as positive control, the pulsed $2 \mathrm{~d}$-stDCs were co-cultured with autologous purified CD4+ T cells (positive selection - Miltenyi Biotec) labeled with CFSE $(2 \mu \mathrm{M})$, after 6 days of co-cultivation, proliferation and expression of CD69 by T cells were assessed by FC and IFN- $\gamma$ was measured by ELISA in culture supernatants. $2 \mathrm{~d}$-stDCs showed to be efficient in inducing proliferation of CD4+ T cells [Figure S3] This figure shows that $50 \%$ of $\mathrm{T}$ cells proliferate in response to the stimulus compared to the unpulsed 2d-stDCs (19.6\%). Besides, 2d-stDCs were able to activate CD4+ cells in response to a nonspecific stimulus such as PHA-M (92.9\% in cell proliferation). A slight difference in CD69 expression when CD4+ T cells were stimulated with $2 \mathrm{~d}$-stDCs pulsed with TT in comparison to negative control (13.4 vs. 8.29\% respectively) and a high CD69 expression when stimulated with PHA-M (43.5\%) were observed. Compared with negative control, TT specific CD4+ T cells produced more IFN- $\gamma$ after 40 hours of co-culture $(10$ and 93pg/mL, respectively, Figure S4A); CD4+ T cells stimulated with $2 \mathrm{~d}$-stDCs pulsed with PHA-M, showed an IFN- $\gamma$ production more than five hundred times greater than the control $(5400 \mathrm{pg} / \mathrm{mL}$, Figure S4A). These results evidence the capacity of $2 \mathrm{~d}$-stDCs to activate and induce proliferation of $\mathrm{CD} 4+\mathrm{T}$ cells in response to specific stimuli.

Similarly, to TT assay, we use a Mixed Leukocyte Reaction (MLR) in the presence of $2 \mathrm{~d}$-stDCs as a model to study the ability of $2 \mathrm{~d}$-stDCs to initiate primary immune responses and to induce the proliferation and CD69 expression in purified CD4+ T cells in an in vitro assay using $2 \mathrm{~d}$-stDCs co-cultured with syngeneic CD4+ $\mathrm{T}$ cells (control) or with CD4+ T cells from different individuals (allogenic) previously labeled with CFSE, during 6 days. As could be shown by FC [FigureS3B], allogenic CD4+ T cells proliferated around $30 \%$ in response to allogeneic stimulation, compared to control, which showed only a $0.7 \%$ of cells growing. The percentage of CD69 positive cells was higher in the allogeneic co-culture (6-9\%), compared to $2.7 \%$ in the control culture [Figure S3B]. In cultured 2d-stDCs with autologous CD4+ T cells no production of IFN- $\gamma$ was evidenced, contrary to cultures containing heterologous lymphocytes with a production of 566 , 338 and $430 \mathrm{pg} / \mathrm{mL}$ of cytokine on each of the tested individuals [Figure S4B]. Altogether the results of proliferation, expression of activation markers and cytokine secretion in cells stimulated with 2d-stDCs let us to argue for a high capacity of these cells to stimulate CD4+ T cells.

To assess the ability of $2 \mathrm{~d}$-stDC to induce an immune response in $\mathrm{CD} 8+\mathrm{T}$ cells specific for tumor associated antigens (TAA), the expansion of CD8+ $\mathrm{T}$ cell precursors labeled by fluorescent tetramers was assessed in healthy donors that express the HLA-A*2:01 allele (determined by SSP-PCR). To do this, TAA peptides restricted to HLA-A2 (Melan-A and NY-ESO 1 at $10 \mu \mathrm{M}$ ) were used to pulse $2 \mathrm{~d}$-stDCs that were co-cultured with autologous CD8+ T cells for 10 days in the presence of IL- 2 and IL-7. After 10 days of cell culture, the T cells were restimulated with 2d-stDC pulsed with peptide for an additional 72 hours and stained with tetramers to identify populations of CD8+ T cells specific for the corresponding TAA. As shown in Figure 4A, the $2 \mathrm{~d}$-stDCs pulsed with peptides Melan-A and NY-ESO1 were 


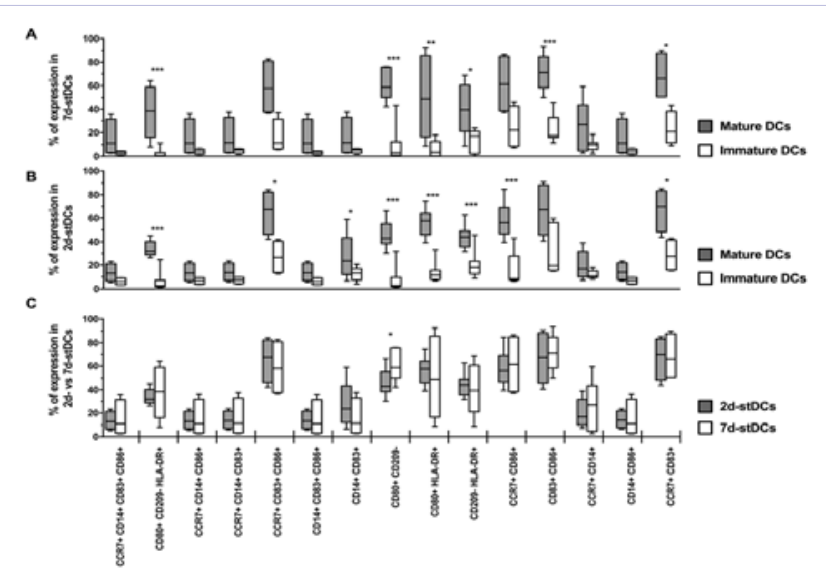

Figure 3: Use of multiple maturation markers for dendritic cells confirms that $\mathbf{2 d - s t D C s}$ and $\mathbf{7 d}$-stDCs have a similar phenotype. Analysis of multiple markers for characterization of dendritic cell maturation (two to four markers) and 2d-stDCs and 7d-stDCs monocyte-derived was analyzed by multiparametric flow cytometry. The statistical analysis of mature (gray box) and immature (white box) cells in 7d-stDCs (A) and 2d-stDCs (B). C. Comparison between mature 7dstDCs (gray box) and 2d-stDCs (white box) derived from adherent and cryopreserved monocytes. The data showed the box and whisker $(95 \%$ and 5\%), Mann-Whitney ${ }^{*} \mathrm{p}<0.05,{ }^{* *} \mathrm{p}<0.01,{ }^{* * *} \mathrm{p}<0.001$.

efficient in the expansion of specific CD8+ precursor cells against these tumor antigens (11 and 5.15\% respectively) compared to control (unstimulated cells, $0.067 \%$ ). Subsequently the cytotoxic capacity of CTLs against tumor antigens were evaluated for CD8+ T cells cultured with $2 \mathrm{~d}$-stDCs pulsed with TAA-A2 and cocultured with DCs pulsed with the corresponding peptide, as a control non-specific peptide of Leishmania major (L. major) and DCs pulsed with nopeptide were used in three healthy donors. To quantitate cytotoxicity the cells were incubated for 5 hours in the presence of anti-CD107a, CD107b and analyzed by flow cytometry. As shown in Figure 4B, a cytotoxic response (CD107a and CD107b positive cells) increased in cells specific against Melan-A antigen (8.36\%) compared to control culture (1.22\%) and culture with peptide L. major (1.31\%). Subsequently, the cytotoxicity (CD107a) of CD8+ T cells in response to different TAAs such as Her-2/neu, NY-ESO1, telomerase and Melan-A in healthy donors expressing HLA-A2 was evaluated; it was observed an increase in positive CD107a cells $(1.3,4.45,1.46$, and $2.74 \%$ respectively) compared to the control culture $(0.43 \%)$ [Figure 4C]. Finally, it was assessed expression of multiple intracellular cytokines (IFN- $\gamma$, TNF- $\alpha$, and IL-2) in CD8+ T cells, after 20 hours of re-stimulation of T cells with mature $2 \mathrm{~d}$-stDC pulsed or not with a cocktail of TAA peptides were determined by flow cytometry multifunctional [Figure 4D] Compared to the control CD8+ T cells and DCs stimulated without peptide, DCs stimulated with peptide cocktail showed increased expression of cells expressing at least one cytokine is $3.5 \%$ vs $2.2 \%$, however, the frequency of $\mathrm{T}$ cells that produce two or more cytokines is higher in cells stimulated with peptides compared to control [Figure 4E], the total percentage of cytokine-producing cells stimulated with peptide that are multifunctional is $3.4 \%$ compared to $0.58 \%$ of unstimulated $\mathrm{T}$ cells. These results support the idea that $2 \mathrm{~d}$-stDCs have the adequate functional capacity to stimulate CD4+ and CD8+ T cells.

\section{Type I alpha DCs that produce IL-12 can be induced and maturated in two days}

Type I alpha DCs, have been recently described by Mailliard et al.[23] using a specific combination of pro-inflammatory cytokines, type I interferons and a TLR3 ligand. So far, the needed time to induce mature alpha DCs (aDCs) has been $7 \mathrm{~d}$ similar to $7 \mathrm{~d}$-stDCs, with few descriptions done in 5 days. To characterize the immune phenotype in $2 \mathrm{~d}$-aDCs we performed a side-byside comparison of the immune phenotype and functional characteristics of immature DCs (iDCs) after treatment for 24 hours with either standard or alpha DC maturation cocktails (2d-stDCs and 2d-aDCs, respectively) based on the methodology of Dauer et al.[21, 28]. Purified monocytes from healthy donors were cultured for 24 hours in the presence of IL-4 (1000 UI/mL) and GM-CSF (750 UI/mL). Subsequently, to induce the maturation of $2 \mathrm{~d}-\mathrm{aDCs}$ or $2 \mathrm{~d}$-stDCs, iDCs were cultured in maturation cocktails containing IL- $1 \beta$, IL- 6 , TNF- $\alpha$, IFN- $\alpha$, IFN- $\gamma$ and Poly I:C [23] or IL-1 $\beta$, IL-6, TNF- $\alpha$ and PGE2 [10], respectively. As shown in Figure 5A, we observed changes in the cell morphology and number of dendrites in response to the maturation stimuli. Consistently in several experiments performed, after 24 hours of maturation the round-shaped iDCs transformed into elongated $2 \mathrm{~d}-\mathrm{aDCs}$ which showed a higher number of thin dendrites than 2d-stDCs. Subsequently, we used multi-parametric Flow Cytometry (FC) to compare the expression of CD83, CD209, CD14, CCR7, CD80, HLA-DR, and CD86 in iDCs, 2d-aDCs, and 2d-stDCs. The standard cytokine cocktail for $2 \mathrm{~d}$-stDCs clearly induces a maturation phenotype in iDCs [Figure 5B], evidenced by the increased expression of CD80, CD83, CD86, CCR7 and HLA-DR and the decreased expression of CD209 and CD14; this phenotype was consistent with the maturation found in Figure 1C and standard studies describing 7d-stDCs $[29,30]$. Notably, treating iDCs with maturation stimuli for 24 hours generates mature $2 \mathrm{~d}$-aDCs with a maturation phenotype similar to that of $2 \mathrm{~d}$-stDCs in terms of increased HLA-DR, CD80, CCR7 and CD83 expression and decreased CD209 and CD14 expression in both cell types [Figure 5B]. One of the main characteristics of Type I alpha DCs is the secretion of IL12p70 (IL-12), a key cytokine for the enhancement of tumor-specific $\mathrm{T}$ cell responses in mouse models [31]. To assess IL-12 secretion levels, we used the CBA kit (BD Biosciences) to quantify the pro-inflammatory cytokines in the supernatants of immature and mature DCs. The results revealed an important difference in the secretion of IL-12, which was only produced in $2 \mathrm{~d}-\mathrm{aDCs}(294 \mathrm{pg} / \mathrm{mL})$ compared with the background levels induced in iDCs and the low levels of 2d-stDCs ( 34.5 and $35.1 \mathrm{pg} / \mathrm{mL}$, respectively; Figure 5C). These results show that the induction of mature aDCs in two days is possible and have a compatible phenotype of a mature DCs similar to the phenotype of 7d-stDCs and 2d-stDCs with the clear advantage of the high production of IL-12.

\section{2d-aDCs activates CD4+ $\mathrm{T}$ cells with a stem-like phenotype that produce IFN- $\gamma$}

Recently Gattinoni et al. described a human stem celllike memory $(\mathrm{scm}) \mathrm{T}$ cell population characterized by a naïve 
phenotype (CD45RA+ CCR7+ CD62-L+ CD28+ CD27+) and the expression of CD95, IL-2R $\beta$, and CXCR3. This population exhibits a superior capacity for proliferation and antitumor activity compared to other memory subsets[32]. Thus, we evaluated the presence and response of $\mathrm{scm} T$ cells after stimulation with either $2 \mathrm{~d}-\mathrm{aDCs}$ or $2 \mathrm{~d}$-stDCs using an in vitro model for infectious diseases. We pulsed each DCs with a combination of Mycobacterium tuberculosis (Mtb) peptides described before[33] that are responsible for a specific in vitro response in tuberculosisinfected donors. For this purpose, we use PBMCs from a latent TB-infected donor (determined by a positive Tuberculin Skin Test - TST > $10 \mathrm{~mm}$, and a positive QuantiFERON TB Gold ${ }^{\circledR}$ test - Cellestis). We induced 2d-aDCs or 2d-stDCs pulsed with or without Mtb peptides. After stimulation for 6 days, the difference in the expansion of naïve, $\mathrm{scm}$, memory or effector CD4+ T cellsub - populations were analyzed on each cell culture through FC subtracting the percentage of each population in peptide pulsed DCs minus the unpulsed DCs [Figure 6A] The IFN- $\gamma$ production in each sub-population (percentage of IFN $-\gamma+\mathrm{CD} 4+\mathrm{T}$ cells pulsed minus unpulsed DCs) demonstrated that the scm compared to central memory (CM), Effector Memory (EM) or terminal effector (EF) $\mathrm{T}$ cell subpopulations produced higher amount of this cytokine only when the culture was stimulated with $2 \mathrm{~d}$-aDCs but not upon stimulation with 2d-stDCs [Figure 6B].

\section{IL-12 produced by $2 \mathrm{~d}$-aDCs favors the expansion of circulating $\mathrm{T}$ follicular helper cells (TFH)}

In the process of differentiation of TFH, the secretion of cytokines such as IL-23, TGF- $\beta$, and IL-12 by APC such as DCs, plays an important role in the differentiation process of naïve $\mathrm{T}$ cells into pre-TFH and from there to GC-TFH or resident memory $\mathrm{TFH}$, with their function as helper cells in antibody production by B lymphocytes[34, 35]. Based on the high production of IL12 by the $2 \mathrm{~d}$-aDCs [Figure $5 \mathrm{C}$ ], we evaluated the ability of these DCs to induce expansion and activation of circulating TFH. To do this, we established an in vitro assay using $2 \mathrm{~d}$-aDCs and $2 \mathrm{~d}$-stDCs derived in PBMCs from healthy donors to characterize by FC the frequency of TFH (CD4+ CXCR5+ PD1+) in CD4+ T cells. Strikingly, we observed in PBMCs from four healthy donors a significant expansion of TFH favored by $2 \mathrm{~d}$-aDCs (mean $2.54 \%$ ) compared with immature DCs (1.29\%) and even 2d-stDCs $(1.64 \%)$ as shown in figure 6C. We also evaluated in the TFH the expression of ICOS and IL-21 as functional markers of these cells in response to stimulation by DCs in combination with TCR activation by beads coupled with anti-CD3/CD28/CD2. In PBMCs from healthy donors, we assessed the expression of ICOS in TFH (CD4+ CXCR5+ PD1+) in response to stimulation of the TCR and we observed a significant increase in the presence of DCs, however 2d-aDCs induce an increased expression of ICOS in TFH (mean 77\%) compared to 2d-iDCs (mean 61\%) and 2d-stDCs (mean 58\%) [Figure 6D left panel]. Finally, we evaluate the intracellular production of IL-21 in the TFH, and as expected, we observe a significant increase in IL-21 MFI in TFH cells when stimulated with $2 \mathrm{~d}$-aDCs (mean 31.28) compared to 2d-iDCs (mean -5.31) and 2d-stDCs (mean 18.7) [Figure 6D right panel]. Together, these results show that $2 \mathrm{~d}$-aDCs can induce IFN- $\gamma$

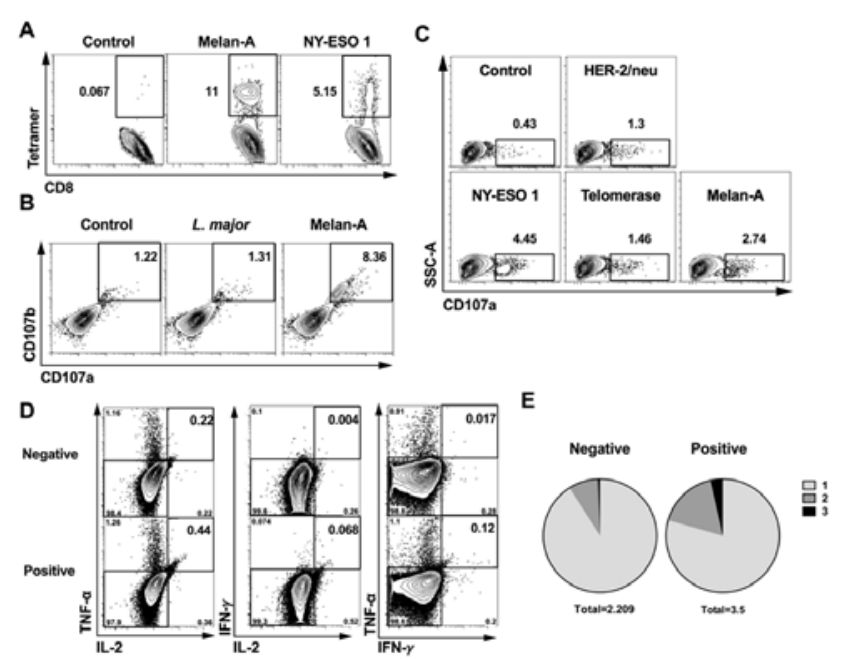

Figure 4: 2d-stDCs expands and activates cytotoxic and multifunctional specific Tumor Associated Antigens (TAAs) CD8+ T cells. A. Tetramer positive CD8+ T cells after 10-day cell culture with Melan-A or NY-ESO 1 peptide-pulsed 2d-stDCs and additional three days with pulsed 2d-stDCs from an HLA-A*02:01 healthy donors. B. Cytotoxicity (CD107a+ CD107b+) of CD8+ T cells one representative plot from 3 different HLA-A*02:01 healthy donors in response to $2 \mathrm{~d}$-stDCs pulsed with Melan-A peptide or controls (unpulsed DCs or non-related peptide L. major). C. Cytotoxicity (CD107a+) of different specific TAAs CD8+ T cells (Melan-A, Her-2/neu, NY-ESO1, and Telomerase) after 10day stimulation with $2 \mathrm{~d}$-stDCs from an HLA-A*02:01 healthy donors. Numbers inside the plots represent the percentage of positive cells. D. Multifunctional CD8+ T cells induced by $2 \mathrm{~d}$-stDCs in response to TAAs peptide pool. CD8+ T cells were stimulated forten days with unpulsed or TAAs peptide pool pulsed 2d-stDCs with a 20 hour boosting with $2 \mathrm{~d}$ stDCs. D. Contour plots of intracellular staining with anti TNF- $\alpha$, IL-2, and IFN- $\gamma$ in negative (Top row) or peptide pulsed (bottom row). E. Pie chart of boolean analysis for cytokine production in negative or TAA peptide pool pulsed 2d-stDCs.

producing CD4+ T cells and they can be used as a tool to evidence the activity of TFH circulating in peripheral blood probably due to its capacity to produce IL-12 in just 48 hours of culture.

\section{Two-day derived DCs induce expansion and activation of tumor-specific $T$ cells in vitro in a breast cancer patient}

In order to characterize antigen specific $\mathrm{T}$ cells present in peripheral blood of cancer patients, it is necessary to employ in vitro systems that allow amplifying TAA specific cells that are probably anergic and present at very low-frequencies. There are different methodologies to increase the number of cells, however, most methodologies require a large volume of blood to obtain the required cells; to address this we use two in vitro systems that not only can expand the antigen specific T cells with a restricted blood volume, but also can evaluate the behavior of naïve repertoire and the generation of memory cells specific to known TAA antigens. The first in vitro system is based on the method described by Martinuzzi et al.[25]. Briefly, iDCs were induced from PBMCs using IL-4 and GM-CSF for 24 hours in the presence of HER2/neu ${ }_{369-377}$ peptide (KIFGSLAFL) and matured 
for an additional 24 hours using $2 \mathrm{~d}$-aDCs or $2 \mathrm{~d}$-stDCs maturation cocktails in the presence of additional HER2/neu peptide ( $5 \mu \mathrm{M})$, followed by stimulation for 6 days [Figure S1A]. The expansion of HER2-specific CD8+ T cells was analyzed through FC using the HER2 dextramer (Immudex), and the memory phenotype (CD45RO vs. CD62-L) was compared between T cells stimulated with iDCs, 2d-aDCs, and 2d-stDCs. We observed an increase in HER2-specific CD8+ T cells in response to stimulation with $2 \mathrm{~d}$-stDCs $(3.6 \%)$ or $2 \mathrm{~d}-\mathrm{aDCs}(3.91 \%)$ compared with unpulsed DCs $(0.94 \%)$ [Figure $7 \mathrm{~A}$ ], suggesting that stimulation with $2 \mathrm{~d}$-stDCs and 2d-aDCs induces the expansion of HER2-specific CD8+ T cells more than 3-fold compared with unpulsed DCs in the breast cancer patient.

Using naïve T-cells cells from the same patient sample, we used a second in vitro system based on the methodology of Moser et al.[27]. Briefly, naïve CD4+ and CD8+ T cells were sorted and cultured for 14 days with $2 \mathrm{~d}$-stDCs or $2 \mathrm{~d}$-aDCs in the presence of HER2/neu KIFGSLAFL peptide or the 20-mer HER2/neu peptide pool, followed by boosting with $2 \mathrm{~d}$-stDCs or $2 \mathrm{~d}$-aDCs for an additional 6 days [Figure S1B]. After stimulation, HER2specific CD8+ T cells showed a 3 to 5 -fold expansion [Figure 7B] compared with unpulsed DCs (from 0.38 to $1.39 \%$ with $2 \mathrm{~d}$-aDCs and 0.41 to $1.98 \%$ with $2 \mathrm{~d}$-stDCs, respectively). To analyze the activation of CD4+ T cells stimulated with 20-mer HER2/neu peptides, we measured CD154 (CD40-L) expression through FC [Figure 7C] and observed that only $2 \mathrm{~d}$-aDCs were able to induce high CD154 expression (MFI 8434 vs. 5190, pulsed vs. unpulsed DCs) compared with CD4+ T cells stimulated with 2d-stDCs (MFI 723 vs. 3543, pulsed vs. unpulsed DCs). We quantified the secretion of IFN- $\gamma$ from the supernatants of CD4+ and CD8+ T cells pulsed and unpulsed with $2 \mathrm{~d}$-aDCs or $2 \mathrm{~d}$-stDCs using a CBA kit (BD biosciences). The results showed higher levels ( $>2$ logs) of IFN- $\gamma$ in CD4+ and CD8+ T cells stimulated with $2 \mathrm{~d}-\mathrm{aDCs}$ (delta

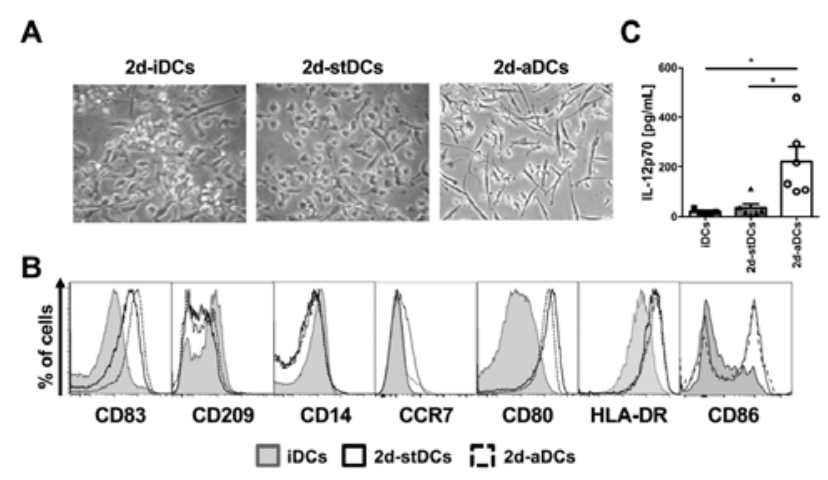

Figure 5: Two-day monocyte-derived dendritic cells using alpha cocktail have a phenotype of mature DCs and secrete high amounts of IL-12p70. A. Microscopic view (40X) of the monocyte-derived DCs 2d-iDCs, 2d-stDCs, and 2d-aDCs (left to right respectively). C. Histograms of the expression of maturation markers CD83, CD209, CD14, CCR7, CD80, HLA-DR, and CD86 of immature dendritic cells (gray histogram), 2d-stDCs (empty histogram), and 2d-aDCs (histogram with dashed line). D. The supernatant quantification of IL-12p70 secreted by 2d-iDCS, 2d-stDCs, and 2d-aDCs. IL-12 secretion is shown in bars (+/SEM), Mann-Whitney * $\mathrm{p}<0.05$. between pulsed minus unpulsed DCs) compared with CD4+ and CD8+ T cells stimulated with 2d-stDCs [Figure 7D]. These results led us to conclude that $2 \mathrm{~d}$-aDCs have a higher capacity than 2d-stDCs to expand and activate specific CD4+ and CD8+ T cells in response to HER2 peptides in a breast cancer patient before treatment.

\section{Discussion}

Since 1973 when Steinman and Cohn gave the name to the cells described by Paul Langerhans "dendritic cells" these cells have been used in different clinical trials as adjuvants to induce specific immune responses against different tumor antigens [1]. From the first study published in 1996 [6], where patients with B-cell lymphoma were vaccinated with autologous DCs (generated in 7 days know as Standard-DCs 7d-stDCs), these cells have been traditionally used as APCs in numerous clinical vaccination protocols [16] with a recently FDA approved therapeutic use for prostate cancer. In 2003, Dauer et al.[21] obtained in only 48 hours of in vitro culture, mature DCs from peripheral blood monocytes of healthy donors, called Fast-DCs (named here $2 \mathrm{~d}$-stDCs). Such cells, upon activation by pro-inflammatory mediators, expressed surface markers of mature DCs, and secrete IL-12p70 when stimulated only with CD40L and IFN- $\gamma$. Additionally, 2d-stDCs were capable of stimulate antigen specific Th1 immune responses [21]. Since then, the role of $2 \mathrm{~d}$-stDCs as APCs has been used in various in vitro assays [36-40] and in some clinical trials with breast cancer patients, who received 4 weekly vaccinations of 2d-stDCs pulsed with Her-2/neuropeptides [41]; in this study, seven of eleven patients showed a marked decrease in the expression of Her-2/neu in tumor specimens. This study demonstrated the potential of $2 \mathrm{~d}$-stDCs in the treatment of early breast cancer.

In order to determine possible changes in the phenotype of the cells in this study we derived 7 and 2 days DCs from monocytes and matured with pro-inflammatory cocktail from of fresh or frozen peripheral blood monocytes, purified by adherence to plastic or by negative selection (rosette formation with red blood cells and CD14 negative cells). In all methods used for their differentiation, DCs $(2 \mathrm{~d}$ and $7 \mathrm{~d})$ stimulated with standard pro-inflammatory cytokines cocktail showed significantly increased expression of CCR7, CD83 and CD80, surface markers important molecules for their performance as professional APCs as compared to unstimulated cells with the cocktail, we even compare the effect of several cytokine combinations including standard cocktail, IFN- $\gamma$, and IL-15 in the maturation phenotype of DCs [Figure S5].

The expression of CD83, a member of the immunoglobulin superfamily, is described as a characteristic marker for mature DCs and correlates with optimal mediated activation of $\mathrm{T}$ cells by DCs[42]. Also, studies in mice have shown that deficiencies in the expression of CD83 reduce the expression of MHC class II on the surface of 25 to $50 \%$ of splenic B cells, DCs, thymic epithelial cells, and peritoneal macrophages[43]. The interaction of the co-stimulatory molecules CD80 and CD86 (B7.1 and B7.2) with $\mathrm{CD} 28$ on $\mathrm{T}$ cells, provides a substantial activation and survival 
of T cells, together with binding MHC/peptide - TCR. Different studies have demonstrated that CD80 is more efficient than CD86 in the activation and proliferation of CD8+ $\mathrm{T}$ cells and the production of IL-12 by DCs [44-46] but the basis of these associations are unknown. Based on the results obtained in this work is interesting to note that CD80 acted as a differentiation marker of mature DCs compared to CD86 which, like HLA-DR, showed a high percentage of expression in mature and immature DCs (both $2 \mathrm{~d}$ and $7 \mathrm{~d}$-stDCs).

CD14 expression was significantly increased in immature and mature cells derived in 2 days compared to 7 days DCs This result contrasts with the observations of Dauer et al.[21] and could be explained by an internalization of CD14 antibody induced by the anti-CD14 beads used in Dauer's study for isolation of monocytes (positive selection). The expression of CD14 in 2d-stDCs suggest that these DCs are similar to dermal interstitial DCs as suggested by the work of Klechevsky et al., who have identified special properties of DCs expressing CD14 which preferentially initiate humoral immunity $[47,48]$.We observed a significant decrease in CD209 (DC-SIGN) in 2d-stDCs in response to maturation cocktail and independently of the purification or preservation process of monocytes. This molecule is often described as a phagocytic receptor with an important role in the acquisition and processing of the antigen on immature DCs,
A

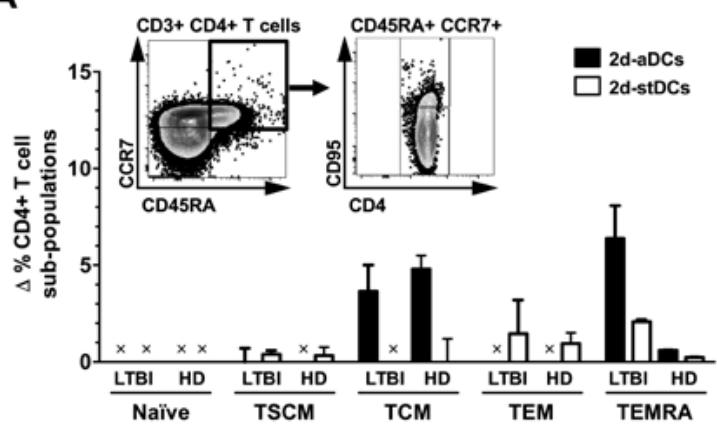

B

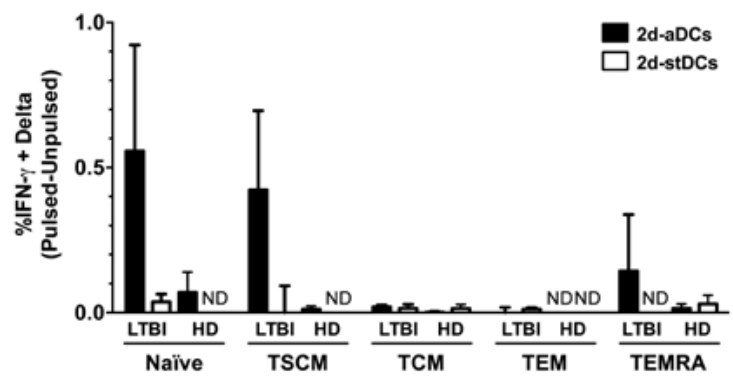

C
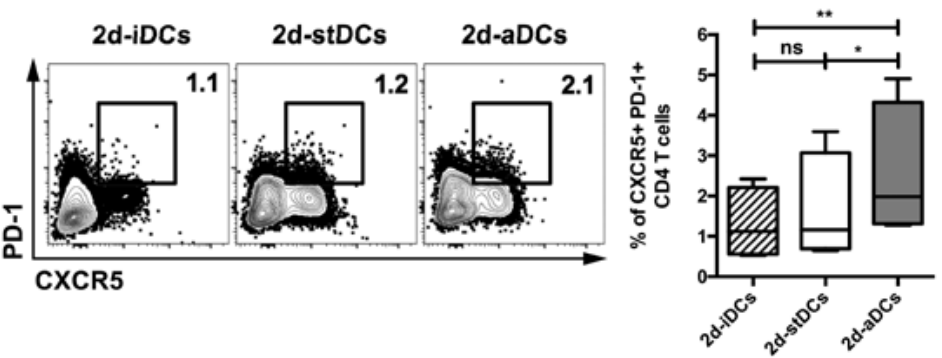

D
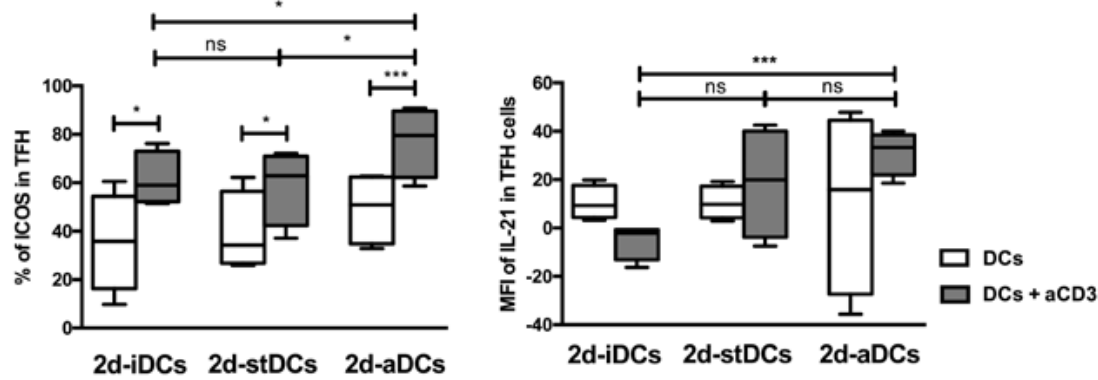

Figure 6: 2d-aDCs induce greater differentiation of memory T cells and expansion of TFH compared with 2d-stDCS. Phenotype characterization of $\mathrm{CD} 4+\mathrm{T}$ cells stimulated with $2 \mathrm{~d}$-aDCs (black bars) or $2 \mathrm{~d}$-stDCs (white bars) pulsed with Mtb peptides minus unpulsed DCs (delta of the percentage) for two days in PBMCs from healthy donors $(n=4)$ or latent tuberculosis infected (LTBI) donors ( $n=4)$, the insert represents contour plots of memory subpopulations (CD45RA vs. CCR7) and CD95+ in naïve cells for TSCM. B. Delta of the percentage of IFN- $\gamma$ producing cells in each memory sub-population quantified in panel A stimulated by 2d-aDCs (black bars) or 2d-stDCs (white bars). C. Quantification of CD4+ T follicular helper (Tfh) cells (CXCR5+ PD1+) in response to 2d-iDCs, 2d-stDCs or 2d-aDCs (left to right panels respectively) and analysis of the quantification of Tfh percentage in healthy donors stimulated with corresponding DCs $(n=4)$. D. Quantification of ICOS + in Tfh cells in response to stimulation with beads coupled to antibodies against CD3 / CD28 / CD2 in combination with DCs. Quantification of Tfh expansion in response to $M t b$ antigens (ESAT-6, QTF peptide pool or H37Rv sonicate) in healthy donors and LTBI donors. Nonparametric $t$ test analysis and paired t-testwere done, ${ }^{*} \mathrm{p}<0.05,{ }^{* * * *} \mathrm{p}<0.0001$. 
characterized with a large capacity of endo-phagocytosis [49, 50]. Since initially described in Human Immunodeficiency Virus (HIV), DC-SIGN has an important role in the immune modulatory response by this lectin in T cells and it has been demonstrated in multiple models of host-pathogen interaction[51, 52], suggesting that some pathogens have immune-evasive properties or immunosuppressant through DC-SIGN.

Overall, the use of fresh or cryopreserved monocytes to derive DCs does not affect the phenotype of the mature DCs derived in two or seven days. These results are of particular importance for immunotherapy considering the possibility of cryopreserving the cells usually obtained in large quantities by leukapheresis, and then used for later generations of DCs.

Besides achieving the expansion of TAA specific $\mathrm{T}$ cell precursors, is important to determine the functionality of these cells, for which CD8+ T cells generated in a specific line of peptide were re-stimulated with $2 \mathrm{~d}$-stDCs pulsed with peptide (target cells) and determined the fusion of the granules with the plasma membrane of the CD8+ T cells that results in the release of perforin and granzyme and surface expression of the membrane glycoproteins associated with the lysosome (LAMPS) CD107a (LAMP-1) and CD107b (LAMP-2). The expression of CD107a and CD107b was determined by FC in CD8+ T cells from four healthy individuals, in response to a given stimulus with $2 \mathrm{~d}$-stDCs pulsed with Melan-A peptide. Similarly, CD8+ T cells generated peptide specific lines NY-ESO1, Her-2/neu, and Telomerase showed expression of $\mathrm{CD} 107 \mathrm{a}$ on the surface by $\mathrm{FC}$, demonstrating the functional capabilities of $2 \mathrm{~d}$-stDCs as antigen-presenting cells.

Mature 2d-stDCs pulsed with peptides were capable to stimulate peptide-specific CD8+ CTLs (specific T cell expansion and cytotoxic functionality by $\mathrm{CD} 107 \mathrm{a} / \mathrm{b}$ expression). Furthermore, substantial production of IFN $-\gamma$ by specific CD4+ T cell in response to Tetanus Toxoid (TT) and in a Mixed Leukocyte Reaction (MLR), suggests that antigen presentation by $2 \mathrm{~d}$-stDCs to T cells can induce the secretion of IL- 12 p70 by the DCs necessary to facilitate the stimulation of Th1 immune responses [Figure S4]. This is important if we consider that IL-12p70 production by DCs has become a key marker of competent DCs that will be used with immunotherapy purposes [53]. Recently, Mailliard et al.[23], described a type I alpha DCs, using a modification of the standard cocktail by adding Type I/II interferons and Poly I:C in order to obtain mature DCs capable of producing high amounts of IL-12p70 and activate Th1 cells responses. But similar to $7 \mathrm{~d}$-stDCs, the type I alpha DCs were produced after seven days of cell culture. These $7 \mathrm{~d}$-aDCS cells are able to load tumor specific antigens and activate breast cancer specific CTL responses [54]. In this work we take the advantage of producing mature DCs in 48 hours and analyzed the functional capability of Type I alpha DCs derived in two days (2d-aDCs) for activation of CD4 and CD8 antigen specific $\mathrm{T}$ cells.

The IL-12p70 production in culture supernatants of mature DCs was determined by CBA. As expected, and according to previous studies where the use of PGE2 in combination with the cocktail of pro-inflammatory cytokines inhibit the production of

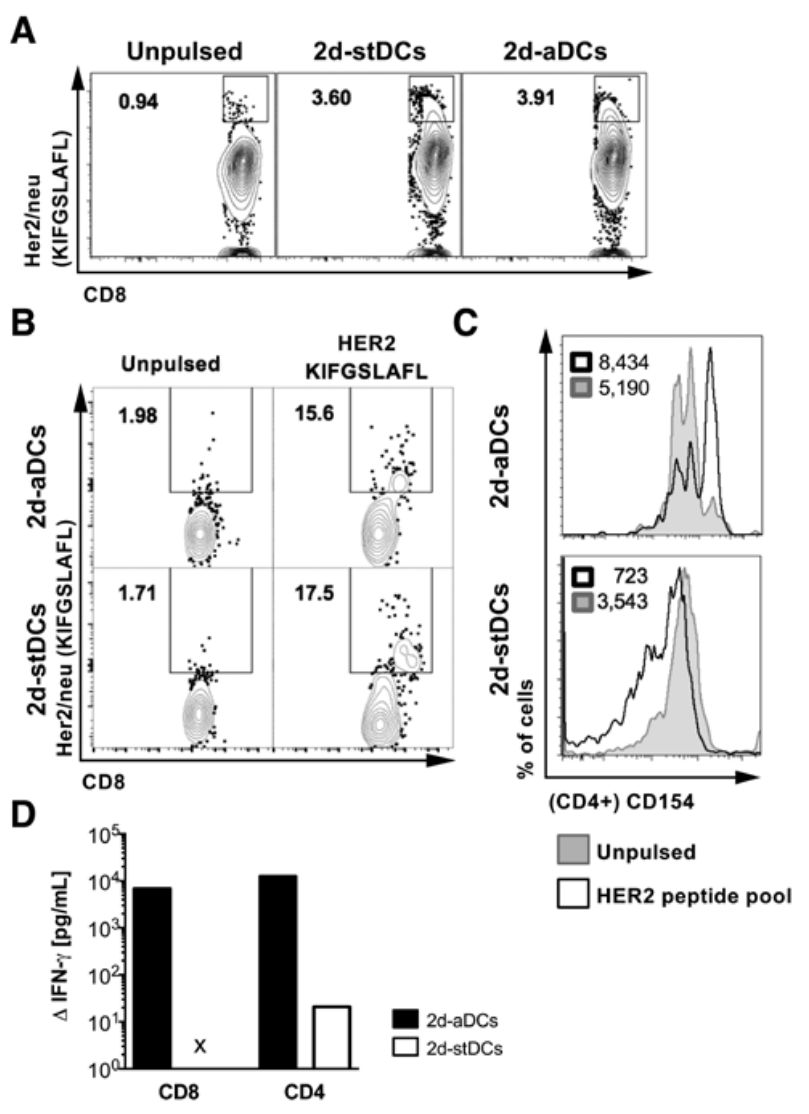

Figure 7: The increase of HER2/neu-specific CD8+ T cells and activation of CD4+ $T$ cells in response to stimulation with $2 \mathrm{~d}$-aDCs in a breast cancer patient. A. Percentage of CD8+ tetramer-positive $\mathrm{T}$ cells in total PBMCs induced with iDCs, $2 \mathrm{~d}$-aDCs or $2 \mathrm{~d}$-stDCs pulsed with HER2 peptide pool for seven days (plot gated on single cells and $\mathrm{CD} 3+\mathrm{T}$ cells) in a breast cancer patient before any treatment. B. Percentage of HER2 tetramer-positive CD8+ T cells after co-culture with $2 \mathrm{~d}$-aDCs or $2 \mathrm{~d}$-stDCs pulsed with HER2 peptide for 14 days and a boosted for six days with $2 \mathrm{~d}$-aDCs or $2 \mathrm{~d}$-aDCs pulsed with HER2 peptide (Unpulsed DCs tinted histogram, HER2 pulsed DCs empty histogram. Gated in singles cells, CD3+/CD8+ T cells). C. MFI of CD154 (CD40-L) on purified CD4+ T cells in response to $2 \mathrm{~d}$-aDCs or 2d-stDCs pulsed with HER2 peptides for 14 days and boosted with $2 \mathrm{~d}$-aDCs or $2 \mathrm{~d}$-aDCs pulsed with HER2 peptides for 6 days (Unpulsed DCs tinted histogram, HER2 peptide-pulsed DCs, empty histogram. Gated in singles cells, CD3+/CD4+ T cells). D. Analysis of IFN $-\gamma$ secretion using a CBA kit (delta of the IFN $-\gamma$ concentration of peptide-pulsed DCs minus unpulsed DCs) in CD4+ and CD8+ $\mathrm{T}$ cell supernatant stimulated with $2 \mathrm{~d}-\mathrm{aDCs}$ or $2 \mathrm{~d}$-stDCs.

IL-12[21, 55], even after additional activation with IFN- $\gamma$ [56], in DCs matured in 2 days with cocktail of pro-inflammatory cytokines produced no substantial amounts of IL-12p70, in contrast to the high production of IL-12 by $2 \mathrm{~d}$-aDCs [Figure $5 \mathrm{C}$ ]. Taking altogether our results, the use of $2 \mathrm{~d}$-aDCs reinforces the benefits of this cytokine combination for differentiation and activation of $\mathrm{T}$ cells. We evaluated the functional capacity of 2d-aDCs and 2d-stDCs for both CD4 and CD8 T cell activation. For Th1 T cell differentiation, we used Mtb. peptides previously 
described to induce IFN- $\gamma$ secretion in immune TB donors $[26,57$, 58]. In the present study, we compared the capacity of peptidepulsed DCs to induce a Th1 phenotype with that of latent-infected donor cells cultured with purified CD4 T cells. After incubation for 6 days, we observed the increased production and secretion of IFN- $\gamma$, TNF- $\alpha$ and IL- 6 compared with unpulsed DCs (data not shown). The analysis of the phenotype of responding cells showed that only stimulation with $2 \mathrm{~d}$-aDCs induced an increase in IFN- $\gamma$ production in scm CD4+ T cells [Figure 6] and not in other memory, naïve or effector sub-populations. This result suggest the interesting possibility that the scm CD4+ T cell memory population is responsible of the IFN- $\gamma$ production in individuals in which Mtb latency has been diagnosed with one of the common IGRA tests available (TB ELISPOT or QuantiFERON TBß).

Another important role of $\mathrm{IL}-12$ is the stimulation of $\mathrm{T}$ follicular helper cells, a specific population of $\mathrm{T}$ cells involved in the antibody production by plasma cells by the release of IL-21. Recent studies show that IL-12 produced by DCs is important to expand and activate TFH cells [35]. We evaluate this possibility using our $2 \mathrm{~d}-\mathrm{aDCs}$, and as expected we found a significant increase in the percentage of circulating CD4+ TFH cells (CXCR5+ and PD1+) in healthy donors, and more importantly, stimulating these cells with anti-CD3/CD28/CD2 beads we were able to induce the activation of TFH determined by the increase ICOS and IL-21 in TFH cells. With these results, we propose that the "in situ" induction of 2d-aDCs in PBMCs it may became a useful tool to study the phenotype and behavior of memory TFH present in peripheral blood and furthermore to evaluate the relationship between the detection of these cells in peripheral blood and the production of antigen specific high antibodies titers by B cells.

The functional capacity of $2 \mathrm{~d}$-aDCs to induce the expansion of CD8 T cells was evaluated in samples from breast cancer patients before chemotherapy to determine the degree of expansion and activation of specific $\mathrm{T}$ cells that recognize different TAAs. The results suggested that $2 \mathrm{~d}-\mathrm{aDC}$ generated from total PBMCs (as described by Martinuzzi et al. [25]) or from purified monocytes as described by Moser et al. [27], [Figure 7A and 7B, respectively] induce the expansion of tumor antigen-specific CD8+ $T$ cells against HER2 peptide and activate the effector function of these cells, evidenced by HER2-dependent IFN- $\gamma$ secretion [Figure 7D] and the expression of CD154 in CD4 T cells obtained from breast cancer patients [Figure $7 \mathrm{C}$ ]. These results suggest that $2 \mathrm{~d}$-aDCs induce the specific response and expansion of $\mathrm{T}$ cells in breast cancer similar to $2 \mathrm{~d}$-stDCs but with an increased Th1 cytokine production. Taken together, the data in the present study suggest that $2 \mathrm{~d}$-stDCs and $2 \mathrm{~d}$-aDCs are efficient short-term APCs and useful in vitro tools to induce TAA specific CD8+ and CD4+ T cell responses (TFH and stem cell like memory T cells) that are suitable for immunotherapy settings, reducing time and cost of DCs development. Further studies should evaluate the combination of $2 \mathrm{~d}$-stDCs and $2 \mathrm{~d}-\mathrm{aDCs}$ for cancer immunotherapy.

\section{Acknowledgments}

This study was supported through funding from the Universidad Nacional de Colombia. DIB, Vicedecanatura de Investigación Universidad Nacional Medical School; funds from a joint grant between Fundación Salud de los Andes, Univesidad Nacional and COLCIENCIAS. The authors express their gratitude to Dr. Fabio Méndez CEO at the Fundación Salud de los Andes (FSA) and to FSA for their generous support. The authors would also like to thank Dr. Bernardo Camacho and to personnel at the Hemocentro Distrital for their kind assistance in obtaining blood buffy coats from volunteers. We are very thankful to Dr. Larry Stern, Dr. Mauricio Calvo-Calle and personnel at their laboratories at the University of Massachusetts for their generous support in providing reagents, infrastructure and knowledge required for building up HLA-A2 tetramers. We thank Dr. Manuel Patarroyo and personnel at the FundaciónInstituto de Inmunología de Colombia (FIDIC) for assistance with peptide synthesis. Finally, our deepest gratitude to patients (especially to the patient FLB001) and healthy volunteers for their generous denotation of blood samples used in this study.

\section{Funding}

This work was funded through Dirección de Investigación de Bogotá (DIB)-HERMES Grants (Numbers 13245, 12543, 33317, 33290, 32181 and 14976) from the Universidad Nacional de Colombia and funds from the joint grant between Fundación Salud de los Andes, Universidad Nacional and COLCIENCIAS (Contract No. 110150227509). DBE was supported by the Fundación Salud de los Andes, Bogotá-Colombia South America.

\section{Author contribution}

Experimental work: DB, and DRTM. Data analysis: DB, DTRM and CP. Wrote the manuscript: DB, DRTM and CP.

\section{References}

1. Steinman, R.M. and Z.A. Cohn, Identification of a novel cell type in peripheral lymphoid organs of mice. I. Morphology, quantitation, tissue distribution. J Exp Med. 1973;137(5):1142-1162.

2. Palucka, K. and J. Banchereau, Cancer immunotherapy via dendritic cells. Nat Rev Cancer. 2012;12(4): 265-277.

3. Draube, A., et al., Dendritic cell based tumor vaccination in prostate and renal cell cancer: a systematic review and meta-analysis. PLoS One. 2011;6(4). doi.org/10.1371/journal.pone.0018801.

4. Avigan, D., Dendritic cells: development, function and potential use for cancer immunotherapy. Blood Rev. 1999;13(1): 51-64.

5. Banchereau, J, et al., Immune and clinical responses in patients with metastatic melanoma to CD34(+) progenitor-derived dendritic cell vaccine. Cancer Res. 2001;61(17): 6451-6458.

6. Hsu, F.J., et al., Vaccination of patients with B-cell lymphoma using autologous antigen-pulsed dendritic cells. Nat Med. 1996;2(1):52-58. doi:10.1038/nm0196-52.

7. Melief, C.J, Cancer immunotherapy by dendritic cells. Immunity. 2008;29(3):372-383.

8. Motta,M.R.,etal.,Generation of dendritic cells from CD14+ monocytes positively selected by immunomagnetic adsorption for multiple myeloma patients enrolled in a clinical trial of anti-idiotype vaccination. Br J Haematol. 2003;121(2):240-250.

9. Osada, T., et al., Dendritic cell-based immunotherapy. Int Rev Immunol. 2006;25(5-6):377-413. 
10. Schuler, G., B. Schuler-Thurner, and R.M. Steinman, The use of dendritic cells in cancer immunotherapy. Curr Opin Immunol. 2003;15(2):138 147.

11. Lenahan, C. and D. Avigan, Dendritic cell defects in patients with cancer: mechanisms and significance. Breast Cancer Res. 2006;8(1):101. doi:10.1186/bcr1375.

12.Pinzon-Charry, A., et al., Numerical and functional defects of blood dendritic cells in early- and late-stage breast cancer. $\mathrm{Br} \mathrm{J}$ Cancer. 2007;97(9):1251-1259. doi:10.1038/sj.bjc.6604018.

13. Curiel, T.J.,et al., Dendritic cell subsets differentially regulate angiogenesis in human ovarian cancer. Cancer Res. 2004;64(16):55355538

14. Fricke, I.and DI. Gabrilovich, Dendritic cells and tumor microenvironment: a dangerous liaison. Immunol Invest. 2006;35(34):459-483

15. Gabrilovich, D., Mechanisms and functional significance of tumourinduced dendritic-cell defects. Nat Rev Immunol. 2004;4(12):941-952.

16. Bloy, N., et al., Trial watch: Dendritic cell-based anticancer therapy. Oncoimmunology. 2014;3(11): e963424

17. Berntsen, A., et al., Therapeutic dendritic cell vaccination of patients with metastatic renal cell carcinoma:a clinical phase 1/2 trial. J Immunother. 2008;31(8): 771-780. doi:10.1097/CJI.0b013e3181833818.

18. Kavanagh, B., et al., Vaccination of metastatic colorectal cancer patients with matured dendritic cells loaded with multiple major histocompatibility complex class I peptides. J Immunother. 2007;30(7): 762-772.

19. Wierecky, J., et al., Immunologic and clinical responses after vaccinations with peptide-pulsed dendritic cells in metastatic renal cancer patients. Cancer Res. 2006;66(11): 5910-5918.

20.Sallusto, F. and A. Lanzavecchia, Efficient presentation of soluble antigen by cultured human dendritic cells is maintained by granulocyte/macrophage colony-stimulating factor plus interleukin 4 and downregulated by tumor necrosis factor alpha. J Exp Med. 1994;179(4): 1109-1118.

21. Dauer, M., et al., Mature dendritic cells derived from human monocytes within 48 hours: a novel strategy for dendritic cell differentiation from blood precursors. J Immunol. 2003;170(8):4069-4076.

22. Dauer, M., et al., FastDC derived from human monocytes within $48 \mathrm{~h}$ effectively prime tumor antigen-specific cytotoxic $T$ cells. J ImmunolMethods. 2005;302(1-2):145-155.

23. Mailliard, R.B., alpha-Type-1 Polarized Dendritic Cells: A Novel Immunization Tool with Optimized CTL-inducing Activity. Cancer Research. 2004;64(17):5934-5937.

24. Jonuleit, H., et al., Pro-inflammatory cytokines and prostaglandins induce maturation of potent immunostimulatory dendritic cells under fetal calf serum-free conditions. Eur J Immunol. 1997;27(12):31353142.

25. Martinuzzi, E., et al., acDCs enhance human antigen-specific T-cell responses. Blood. 2011;118(8): 2128-2137. doi:10.1182/ blood-2010-12-326231.

26. Goletti, D., et al., Region of difference 1 antigen-specific CD4+ memory T cells correlate with a favorable outcome of tuberculosis. J Infect Dis. 2006;194(7):984-992.

27. Moser, J.M., et al., Optimization of a dendritic cell-based assay for the in vitro priming of naive human CD4+ T cells. J Immunol Methods.

\section{0;353(1-2):8-19. doi:10.1016/j.jim.2009.11.006}

28. Dauer, M., et al., FastDC derived from human monocytes within $48 \mathrm{~h}$ effectively prime tumor antigen-specific cytotoxic $T$ cells. Journal of Immunological Methods. 2005;302(1-2):145-155.

29. Sallusto, F. and A. Lanzavecchia, Understanding dendritic cell and T-lymphocyte traffic through the analysis of chemokine receptor expression. Immunol Rev. 2000;177:134-140.

30. Obermaier, B., et al., Development of a new protocol for 2-day generation of mature dendritic cells from human monocytes. Biol Proced Online. 2003;5:197-203. doi:10.1251/bpo62.

31. Nishioka, Y., et al., Induction of systemic and therapeutic antitumor immunity using intratumoral injection of dendritic cells genetically modified to express interleukin 12. Cancer Res. 1999;59(16): 40354041.

32. Gattinoni, L., et al., A human memory $T$ cell subset with stem cell-like properties. Nat Med. 2011;17(10): 1290-1297. doi: 10.1038/nm.2446.

33.Goletti, D., et al., Selected RD1 peptides for active tuberculosis diagnosis: comparison of a gamma interferon whole-blood enzymelinked immunosorbent assay and an enzyme-linked immunospot assay. Clin Diagn Lab Immunol. 2005;12(11): 1311-1316. doi:10.1128/ CDLI.12.11.1311-1316.2005

34.Vinuesa, C.G., et al.,Follicular Helper T Cells. Annu Rev Immunol. 2016;34:335-368. doi:10.1146/annurev-immunol-041015-055605.

35. Schmitt, N., et al., Human dendritic cells induce the differentiation of interleukin-21-producing $T$ follicular helper-like cells through interleukin-12. Immunity. 2009;31(1):158-169. doi:10.1016/j. immuni.2009.04.016

36. Jarnjak-Jankovic, S., et al., A full scale comparative study of methods for generation of functional Dendritic cells for use as cancer vaccines. BMC Cancer. 2007; 7:119.

37. Ramadan, G., et al., Generation of cytotoxic T cell responses directed to human leucocyte antigen Class I restricted epitopes from the Aspergillus f16 allergen. Clin Exp Immunol. 2005;140(1): 81-91.

38. Dauer, M., et al., Combined use of toll-like receptor agonists and prostaglandin E(2) in the FastDC model: rapid generation of human monocyte-derived dendritic cells capable of migration and $I L-12 p 70$ production. J Immunol Methods. 2008;337(2):97-105. doi:10.1016/j. jim.2008.07.003.

39. Zhu, F., et al., Stimulation by means of dendritic cells followed by EpsteinBarr virus-transformed $B$ cells as antigen-presenting cells is more efficient than dendritic cells alone in inducing Aspergillus f16-specific cytotoxic T cell responses. Clin Exp Immunol. 2008;151(2):284-296.

40. Guan, X., et al., A novel, rapid strategy to form dendritomas from human dendritic cells and hepatocellular carcinoma cell line HCCLM3 cells using mature dendritic cells derived from human peripheral blood CD14+ monocytes within 48 hours of in vitro culture. World J Gastroenterol. 2004;10(24):3564-3568.

41. Czerniecki, B.J., et al., Targeting HER-2/neu in early breast cancer development using dendritic cells with staged interleukin-12 burst secretion. Cancer Res. 2007;67(4):1842-1852.

42. Kruse, M., et al., Inhibition of CD83 cell surface expression during dendritic cell maturation by interference with nuclear export of CD83 $m R N A$. J Exp Med. 2000;191(9):1581-1590.

43. Kuwano, Y., et al., CD83 influences cell-surface MHC class II expression on $B$ cells and other antigen-presenting cells. Int Immunol. 2007;19(8):977-992. 
44. Fields, P.E., et al., B7.1 is a quantitatively stronger costimulus than $B 7.2$ in the activation of naive CD8+ TCR-transgenic T cells. J Immunol. 1998;161(10):5268-5275.

45. Gajewski, T.F., B7-1 but not B7-2 efficiently costimulates CD8+T lymphocytes in the P815 tumor system in vitro.J Immunol. 1996;156(2): 465-472.

46. Pejawar-Gaddy, S. and M.A. Alexander-Miller, Ligation of CD80 is critical for high-level CD25 expression on CD8+ T lymphocytes. J Immunol. 2006;177(7): 4495-4502. doi:10.4049/jimmunol.177.7.4495.

47. Klechevsky, E., et al., Understanding human myeloid dendritic cell subsets for the rational design of novel vaccines. Hum Immunol. 2009;70(5):281-288. doi:10.1016/j.humimm.2009.02.004.

48. Klechevsky, E., et al., Functional specializations of human epidermal Langerhans cells and CD14+ dermal dendritic cells. Immunity. 2008;29(3):497-510. doi:10.1016/j.immuni.2008.07.013.

49. Geijtenbeek, T.B., et al., Self- and nonself-recognition by C-type lectins on dendritic cells. Annu Rev Immunol. 2004;22:33-54.

50. Kerrigan, A.M. and G.D. Brown, C-type lectins and phagocytosis Immunobiology. 2009;214(7):562-575. doi: 10.1016/j. imbio.2008.11.003.

51. den Dunnen, J., S.I. Gringhuis, and T.B. Geijtenbeek, Innate signaling by the C-type lectin DC-SIGN dictates immune responses. Cancer Immunol Immunother. 2009;58(7):1149-57.doi:10.1007/s00262-008-0615-1.
52.Wu, L. and V.N. KewalRamani, Dendritic-cell interactions with HIV: infection and viral dissemination. Nat Rev Immunol. 2006;6(11):859868.

53. DeBenedette, M.A., et al., Priming of a novel subset of CD28+ rapidly expanding high-avidity effector memory CTL by post maturation electroporation-CD40L dendritic cells is IL-12 dependent. J Immunol. 2008;181(8):5296-5305.

54.Park, M.H., et al., Alpha-Type 1 Polarized Dendritic Cells Loaded with Apoptotic Allogeneic Breast Cancer Cells Can Induce Potent Cytotoxic T Lymphocytes against Breast Cancer. Cancer Res Treat. 2011;43(1): 56-66. doi:10.4143/crt.2011.43.1.56

55.van der Pouw Kraan, T.C., et al., Prostaglandin-E2 is a potent inhibitor of human interleukin 12 production. J Exp Med. 1995;181(2):775-779.

56. Dauer, M., et al., IFN-alpha promotes definitive maturation of dendritic cells generated by short-term culture of monocytes with GM-CSF and IL-4. J Leukoc Biol. 2006;80(2):278-286.

57.Petruccioli, E., et al., IFNgamma/TNFalpha specific-cells and effector memory phenotype associate with active tuberculosis. J Infect. 2013;66(6): 475-486. doi:10.1016/j.jinf.2013.02.004.

58. Goletti, D., et al., Accuracy of an immune diagnostic assay based on RD1 selected epitopes for active tuberculosis in a clinical setting: a pilot study. Clin Microbiol Infect. 2006;12(6):544-550.DOI: doi. org/10.1111/j.1469-0691.2006.01391.x. 
A

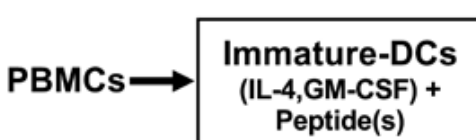

B

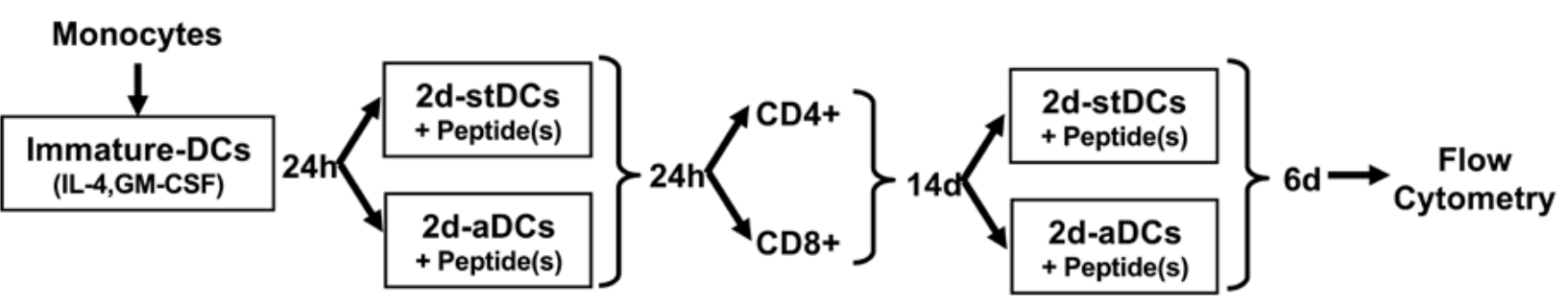

Figure S1: Flowchart of the methodology used for cell culture with 2d-aDCs and 2d-stDCs. A. Methodology for 2d-aDC and 2d-stDC induction in total PBMCs (based on the method of Martinuzzi et al.[25]). For flow cytometry, 10^6 PBMCs were stimulatedduring $24 \mathrm{~h}$ with IL-4 and GMCSF $(750 \mathrm{IU} / \mathrm{mL}$ and $1000 \mathrm{IU} / \mathrm{mL}$, respectively) and with pro inflammatory cytokines and TLR ligands for the corresponding DCs with or without peptide(s) for six days. B. T cell stimulation scheme based on the methodology of Moser et al.[27]. Briefly 2d-aDCs and 2d-stDCs were derived from monocytes and cultured with purified CD4+ or CD8+ T cells for 14 days and re-stimulated for 6 days with peptide $(5 \mu \mathrm{M})$-pulsed $2 \mathrm{~d}$-aDCs or $2 \mathrm{~d}$ stDCs. Flow cytometry was performed using $\mathrm{T}$ cells for immuno-phenotyping and supernatants for cytokine quantification by CBA. 
A

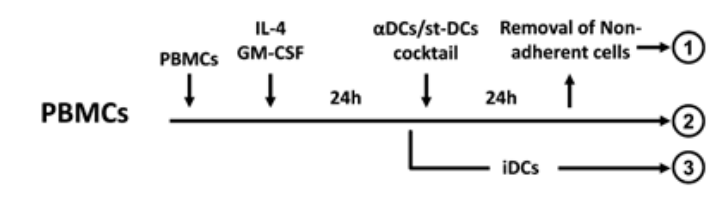

B
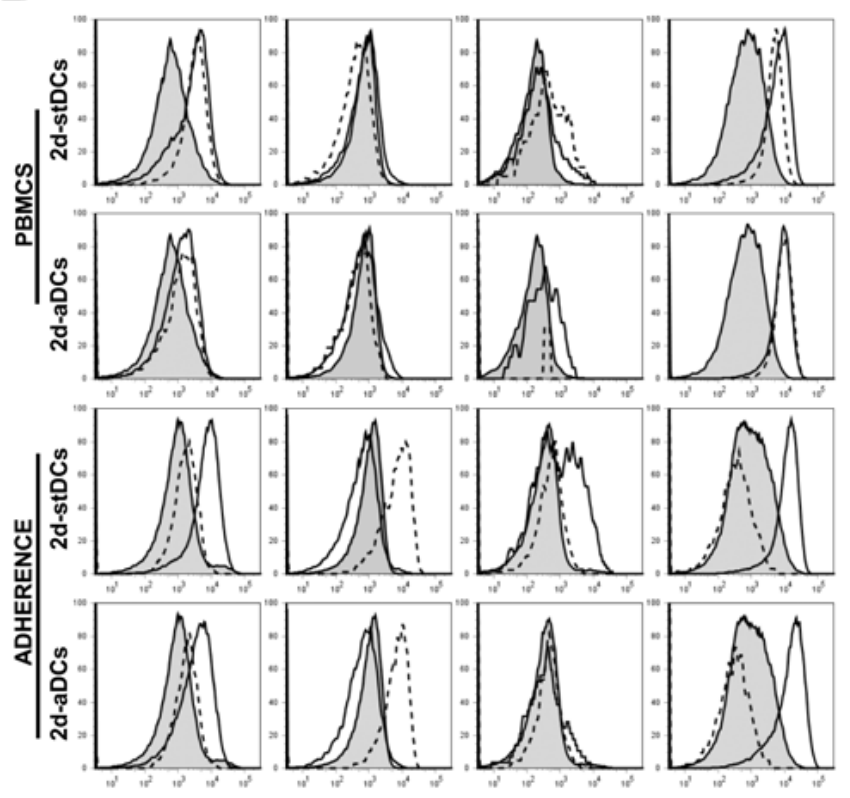

CD14

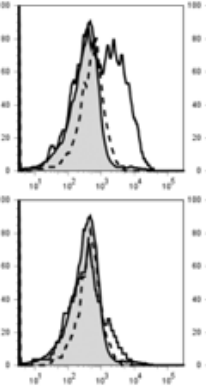

CCR7

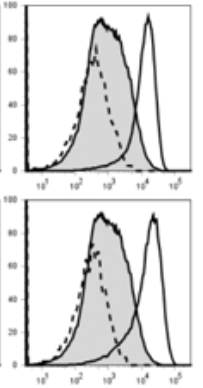

CD80

Figure S2: Maturation profile of DCs obtained through plastic adhesion or direct maturation in PBMCs. A. In vitro DCs generation scheme. The top scheme (PBMCs) DCs were induced in total cells with either aDC or stDC cytokine cocktails, (1) non-adherent cells exposed to maturation cocktail, (2) mature DCs adhered to plastic and (3) immature DCs. The bottom scheme represents the adherent method to induce mature DCs, (4) mature 2d-aDCs or 2d-stDCs derived from adherent cells, (5) iDCs from adherent cells (exposed only to IL-4 and GM-CSF) and (6) non-adherent cells without cytokine exposure. B. Expression profile (CD83, CD14, CCR7, and CD80) in iDCs (tinted histogram), adherent (empty histograms) and non-adherent cells (dashed histograms) derived from total PBMCs (top row) as described in panel A or adherence method (bottom rows) with aDCs or stDCs cytokine cocktail. All histograms were gated on single cells (FSC-A vs. FSC-H) and DCs based on FSC-A vs. SSC-A (CD11c-positive cells). 
A

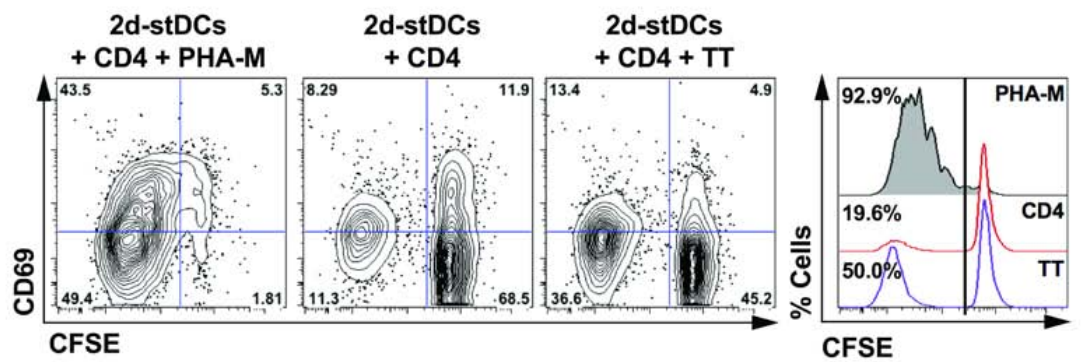

B

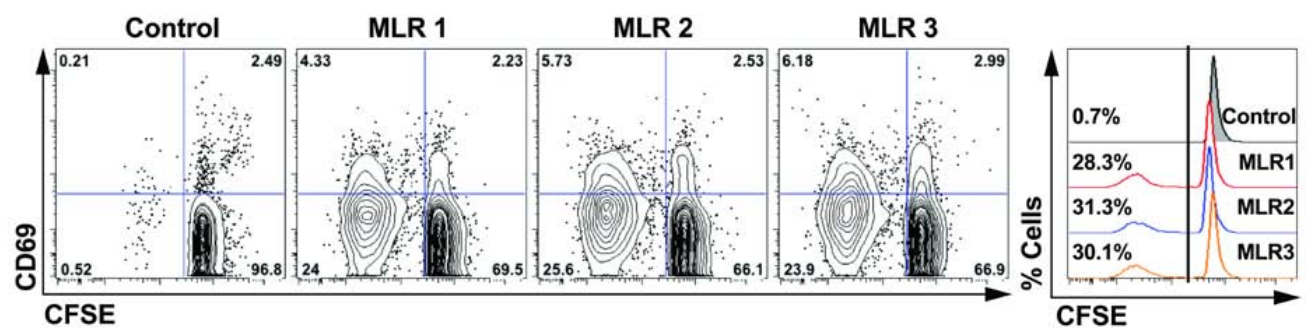

Figure S3: 2d-stDCs properly induce CD4+ T cell activation and proliferation. A. CFSE labeled CD4+ T cells were stimulated with unpulsed or pulsed 2d-stDCs with tetanus toxoid (TT) or PHA-M during 5 days. Contour plots of CFSE vs CD69 in CD4+ T cells (left panels) and overlapping histograms of CFSE dilution in CD4+ T cells (right panel). Proliferating and activated CD4+ T cells (CFSE- CD69+) in presence of PHA-M (positive control 43.5\%), unpulsed (negative control 8.29\%) and TT (13.4\%) pulsed 2d-stDCs. Histograms (right panel) show dilution of CFSE in CD4+ T cells with PHA-M (92.9\%), unpulsed (19.6\%) and TT pulsed 2d-stDCs (50\%). B. Activation and proliferation analysis of CD4+ T cells in a Mixed Leukocyte Reaction (MLR) with 3 different healthy donors in co-culture with 2d-stDCs, contour plots of CFSE vs CD69 in CD4+ T cells (left panels) and overlapping histograms of CFSE dilution in CD4+ T cells. Proliferating and activated CD4+ T cells (CFSE- CD69+) in presence of autologous CD4+ T cells $(0.21 \%)$ or 3 different donors $(4.33,5.73$ and $6.18 \%)$ and proliferation with autologous CD $4+\mathrm{T}$ cells $(0.7 \%)$ or with 3 different donors $(28.3,31.3$ and $30.1 \%)$. 
A

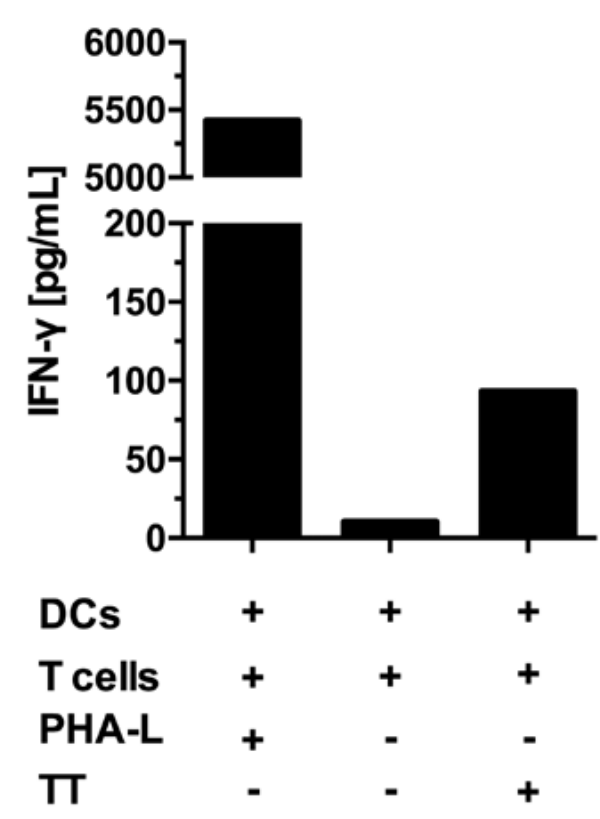

B

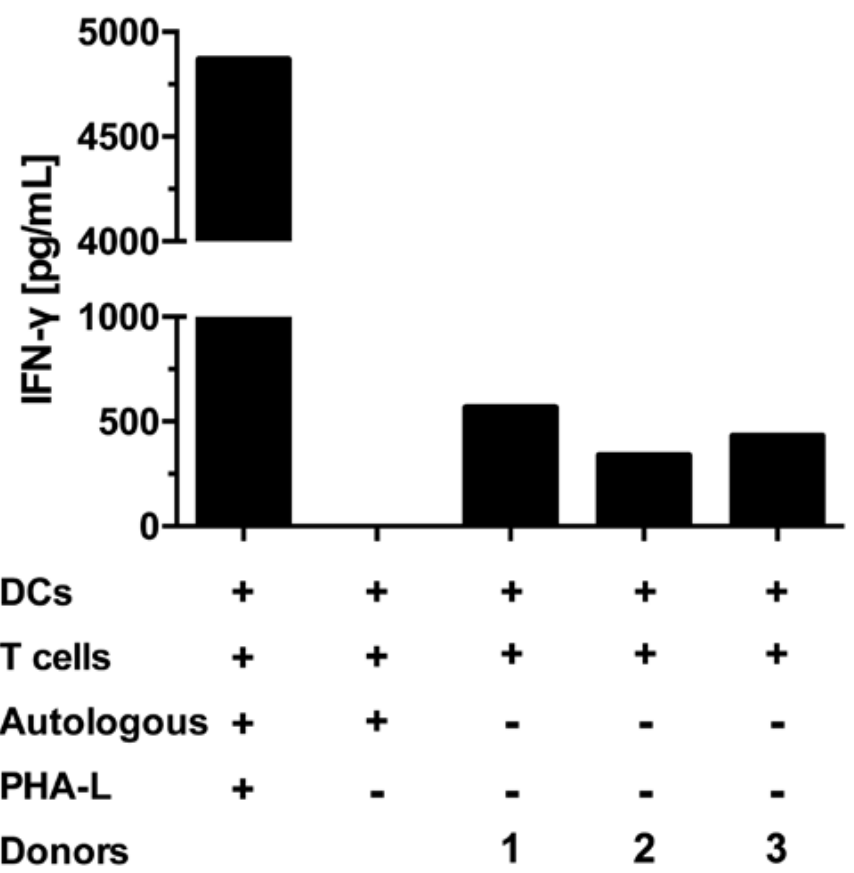

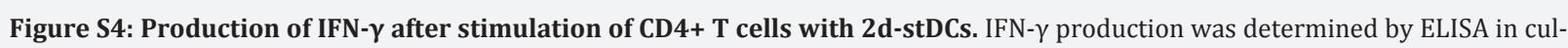
ture supernatants after 40 hours of in vitro cell culture. A) The culture of CD4+ T cells stimulated with DCs pulsed with $2 \mathrm{~d}$-stDCs PHA-L (5 $\mu \mathrm{M})$, alone or with ten $\mu \mathrm{g} / \mathrm{mL}$ of TT. B) Mixed Leukocyte Reaction (MLR) with 2d-stDCs in coculture with CD4+ T cells from 3 different healthy individuals, as positive control autologous CD4+ T cells, stimulated with PHA-L ( $5 \mu \mathrm{M})$ or without PHA-L as a negative control. 


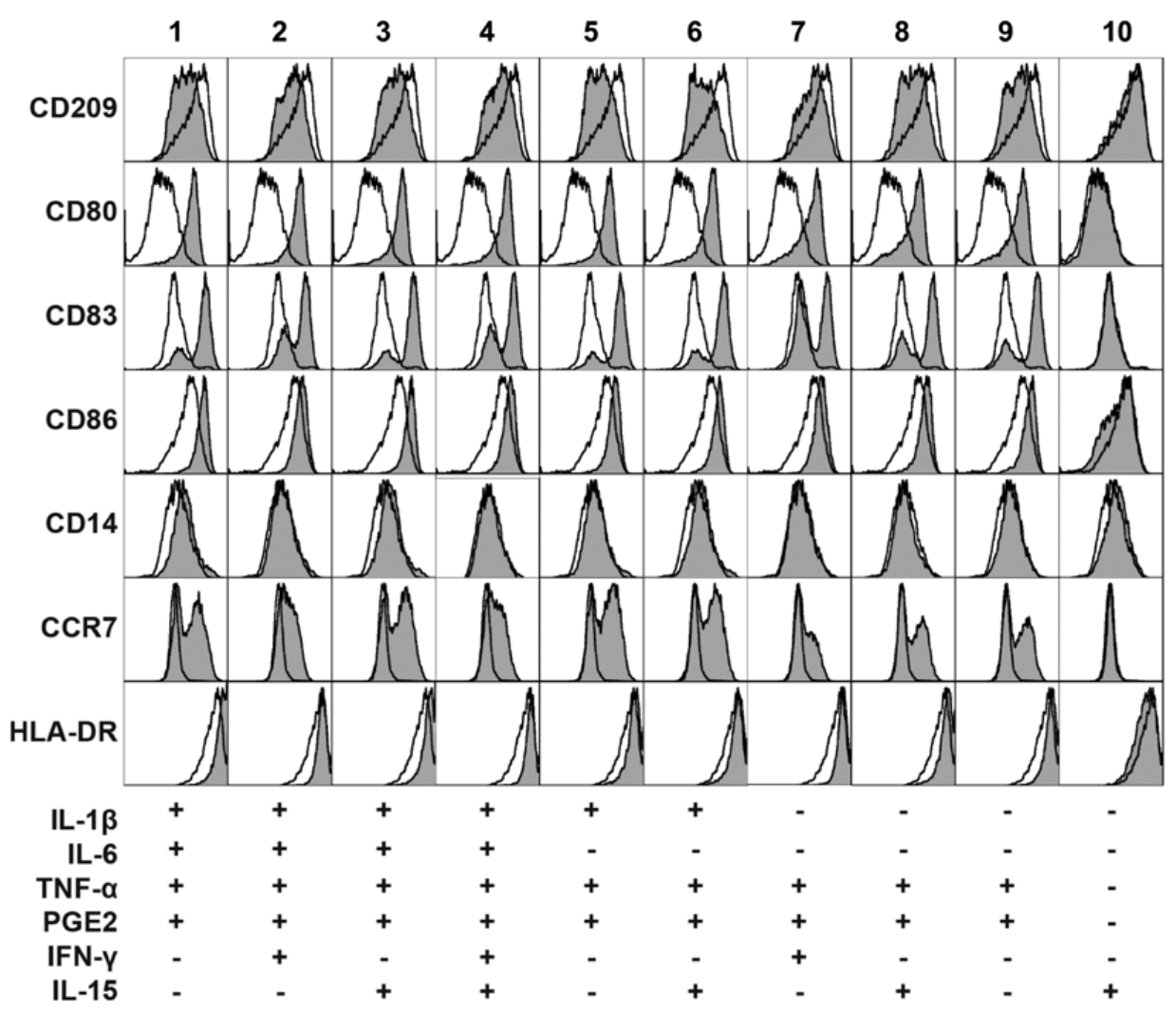

Figure S5: Different cytokine combinations can alter the phenotype of mature DCs. Ten different cytokine combinations were analyzed using Il-1 $\beta$, IL-6, TNF- $\alpha$, PGE-2, IFN- $\gamma$ and IL-15. Phenotype analyses were done using flow cytometry to determine the expression of CD209, CD80, CD83, CD86, CD14, CCR7 and HLA-DR, after two days of maturation culture. Empty histograms represent immature DCs (IL-4 and GM-CSF) and filled histograms represent mature DCs with corresponding cytokine combination (1-10). 